\title{
Wave propagation in presence of oscillators on the free surface
}

\author{
Claude Boutin *, Pierre Roussillon \\ Ecole Nationale des Travaux Publics de l'Etat, Laboratoire Géomatriaux, Department Genie Civil et Batiment, \\ URA CNRS 1652, 69518 Vaulx-en-Velin Cedex, France
}

\begin{abstract}
This article is devoted to wave propagation in presence of a periodic distribution of oscillators (3D sprung-damped mass) on the surface of an elastic medium. The case of an elastic half space loaded by quasi-periodic surface forces is studied at first. The equivalent boundary conditions at the macro-scale are derived at the first and second order, by assuming the existence of a boundary layer and using a two-dimensional homogenisation method. When the force distribution results from attached oscillators, it is shown that boundary conditions can be expressed in terms of equivalent surface impedance at the first order, with local and non-local correctors at the second order. These results are used to study wave refraction by an oscillator layer. The main phenomena - atypical redistribution of mode and mode conversion, frequency range of efficiency, characteristic time of response - are identified. The first correctors are determined analytically for plane waves of oblique incidence. Finally the validity range of the modelling is discussed.
\end{abstract}

Keywords: Wave propagation; Boundary layer; Homogenisation; Resonant surface; Surface scattering

\section{Introduction}

The aim of this paper is to investigate the effect a "resonant surface" on the wave field in an elastic homogeneous half space. Resonant surface means a "layer" on the "free" surface made of a distribution of linear oscillators. This question is encountered:

- at large scale in geophysics, for instance to investigate the city influence on the seismic response $[18,8,10]$,

- in geotechnical surface wave survey, to identify whether elements as trees, houses, vehicles,..., dispatched on the surface may modify the free field assumption in a given frequency range,

- in acoustics and ultrasound auscultation to clarify how an extremely corrugated surface should be taken into account.

\footnotetext{
* Corresponding author.

E-mail address: claude.boutin@entpe.fr (C. Boutin).
} 
In this study, the distribution of oscillators that constitutes the resonant surface shows some spatial invariance in plane, or, in other words, is characterised by a representative surface element (RSE) containing several oscillators. More precisely, a 2D periodic repetition of the same RSE pattern of surface $\Sigma_{0}$ and characteristic size $l$ is assumed. Even in such a regular case, the response of the elastic half space coupled with the oscillators includes multiple crossed interactions and the exact treatment is extremely complex $[7,16]$. However, the complexity is largely reduced when the surface motion shows a characteristic size of variation significantly larger than the characteristic $\Sigma_{0}$-size, $l,[6]$. This condition of scale separation [1], systematically assumed in this study, is satisfied at sufficiently low frequency so that the wavelength $\lambda$ (macro-scale) is greater than $l$ (micro-scale). These assumptions allow to use the framework of two scale asymptotic homogenisation method classically used for 3D periodic media, [12]. In the present case, the periodicity is restricted to the two dimensions of the interface plane. The method allows to derive the behaviour at macro-scale, from phenomena at micro-scale. The leading order gives the equivalent continuous description with an accuracy of the order of the micro to macro-scale ratio. When the scale separation is not very large, the description is improved by considering the next order terms.

The investigated phenomena is close to the so called "fuzzy structure" effect, i.e. the modification of the vibration modes of a main structure by a multitude of small substructures attached to it. Despite the differences in the methods and problems in consideration, the results obtained from "fuzzy structure" probabilistic $[14,15]$ or deterministic theory [17] present some similarities with those derived from the asymptotic homogenisation method. The main improvement provided by this latter lies in the rigorous derivation of both leading effects and first correctors for any kind of wave field that satisfies the scale separation. This condition is explicitely formulated and defines unambigousely the validity domain of the modelling. Asymptotic approaches were already applied to wave refraction on regularly corrugated surfaces — see for instance $[13,3,11]$ - however, the imperfections are neither sufficiently sharp nor sufficiently slender to act as resonators. The key point of the present analysis is to include the amplification phenomena linked to the resonance of the oscillators. This allows to design "resonant surfaces" (as those described in the conclusion) in the purpose to modify in a prescribed way the reflection properties.

The paper begins with a presentation of the homogenisation method that enables to treat two-dimensional heterogeneity distributions by introducing a boundary layer, Sanchez-Palencia [12]. The application to an elastic half space loaded by quasi-periodic surface forces yields the macro-scale boundary conditions at the leading and next orders. When the force distribution results from attached oscillators, it is shown that boundary conditions can be expressed in terms of equivalent surface impedance at the leading order, with local and non-local correctors at the next order. These results are then used to study the perturbation of waves field in presence of a resonant surface. The main features of the phenomena-atypical redistribution of mode and mode conversion, frequency range of efficiency, characteristic time of response-are identified for oblique incident plane waves. Further, the first correctors are explicitely determined. Finally the validity range of the modelling is discussed.

\section{Statement of the problem-homogenisation method}

Consider a resonant surface made of a $\Sigma_{0}$-periodic distribution of linear oscillators. These oscillators lie on top plane surface $\Gamma$ of an homogeneous elastic half space characterised by its elastic tensor $\mathbf{C}$ and its density $\rho$ (viscoelastic media may be similarly treated by considering complex moduli).

Our objective is to study in this system the propagation of small amplitude harmonic waves of frequency $f=\omega / 2 \pi$, assuming a clear scale separation between the characteristic size $l$ of the period $\Sigma_{0}$ and the wavelength in the medium:

$$
\epsilon=2 \pi l / \lambda \ll 1
$$

Furthermore, wave frequency and oscillator's natural frequencies are assumed to be of the same order of magnitude.

The system linearity ensures that all the elements oscillate at the imposed frequency $f$. The oscillators set in motion by the waves induce on the surface $\Gamma$ of the medium an heterogeneous distribution of surface forces, $\mathbf{t} \exp [\mathrm{i} \omega t]$, (t has the dimension of a stress) (Fig. 1). 


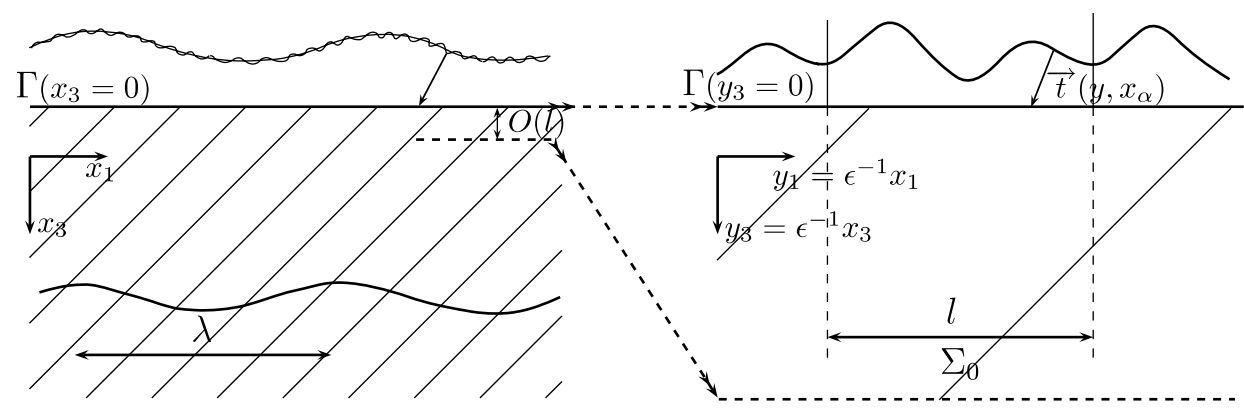

Fig. 1. Half-space loaded by quasi-periodic surface forces. Left: macroscopic description. Right: microscopic description of the boundary layer.

Without specifying the surface forces more precisely (this will be achieved in Section 4), it is clear that

- at the micro-scale, i.e. $\Sigma_{0}$-scale, the force distribution is locally $\Sigma_{0}$-periodic,

- the distribution may also vary at the macro-scale, i.e. the wavelength scale.

\subsection{Multi-scale expansions}

In order to describe the variations at both scales, two space variables linked by the scale ratio $\epsilon$ will be used, namely the macro-variables $\mathbf{x}$ and the micro-variables $\mathbf{y}$, with $\mathbf{y}=\epsilon^{-1} \mathbf{x}$. Following the homogenisation procedure [2,12], the physical quantities are expressed in the form of asymptotic expansions in power of $\epsilon$.

The local 2D periodicity of the surface forces enforces the same 2D local periodicity of the physical quantities in the medium. However, the sources of these small scale variations being located on the top surface only, it is expected that far from the boundary, the small scale variations vanish, while the large scale variations remain. Such situation can be described by introducing a boundary layer in the vicinity of the surface, as proposed by Sanchez-Palencia [12] for thermal transfer conditions and Bouchitté et al. [4], for nonlinear contact problems.

These assumptions lead to postulate a solution on the form defined hereafter. Conveniently, the $\mathbf{x}$ and $\mathbf{y}$ frames are chosen in such a way that $\Gamma$ (the interface between the elastic medium and the oscillator layer) is defined by $x_{3}=0,\left(y_{3}=0\right)$, with outward normal $-\mathbf{e}_{3}$. Throughout this paper, Latin index runs from 1 to 3 , Greek index runs from 1 to 2 , and $\mathbf{x}_{\alpha}$ denotes the projection of $\mathbf{x}$ on $\Gamma$.

\subsection{Macro-field far from the surface}

Far from the surface, only the macro-variables are relevant. The usual form of the elastodynamics equations then applies on the expanded quantities (here and in the following, the time dependence, exp[i $\omega t]$ is systematically omitted):

$$
\mathbf{u}(\mathbf{x})=\sum_{\mathbf{i}=\mathbf{0}}^{\infty} \epsilon^{\mathrm{i}} \mathbf{u}^{\mathrm{i}}(\mathbf{x}), \quad \sigma(\mathbf{x})=\sum_{\mathrm{i}=\mathbf{0}}^{\infty} \epsilon^{\mathrm{i}} \sigma^{\mathrm{i}}(\mathbf{x})
$$

with

$$
\sigma(\mathbf{x})=\mathbf{C}: \mathbf{e}_{\mathbf{x}}(\mathbf{u}), \quad \text { and } \quad \operatorname{div}_{\mathbf{x}}(\sigma)=-\rho \omega^{2} \mathbf{u}
$$

The index $\mathbf{x}$ (or $\mathbf{y}$ ) specifies the derivation variable, and $\mathbf{u}, \mathbf{e}(\mathbf{u}),\left(e_{i j}=\left(u_{i, j}+u_{j, i}\right) / 2\right), \sigma$, respectively denote the displacement, the strain tensor and the stress tensor.

This macro-field does not match the small scale variations of the surface forces. We shall see later that the boundary conditions to be respected by the macro-field on $\Gamma$ are provided by the homogenisation procedure. 
In order to fit the conditions on the surface, a boundary layer (BL) field (denoted by ${ }^{\star}$ ) is added to the macro-field. This additional BL field, confined near the surface, varies at the micro-scale, i.e. according to $\mathbf{y}$, and at the macro-scale according to $\mathbf{x}_{\alpha}$. Keeping in mind that in the system of two space variables, the spatial derivative becomes $\partial / \partial x+\epsilon^{-1} \partial / \partial y$, the elastodynamics equations take the following form in the boundary layer:

$$
\begin{aligned}
& \sigma(\mathbf{x})+\sigma^{\star}\left(\mathbf{x}_{\alpha}, \mathbf{y}\right)=\mathbf{C}:\left(\epsilon^{-\mathbf{1}} \mathbf{e}_{\mathbf{y}}+\mathbf{e}_{\mathbf{x}}\right)\left(\mathbf{u}+\mathbf{u}^{\star}\right) \\
& \left(\epsilon^{-\mathbf{1}} \operatorname{div}_{\mathbf{y}}+\operatorname{div}_{\mathbf{x}}\right)\left(\sigma+\sigma^{\star}\right)=-\rho \omega^{2}\left(\mathbf{u}+\mathbf{u}^{\star}\right)
\end{aligned}
$$

and from the Eqs. (3) governing the macro-field:

$$
\begin{aligned}
& \sigma^{\star}\left(\mathbf{x}_{\alpha}, \mathbf{y}\right)=\mathbf{C}:\left(\epsilon^{-1} \mathbf{e}_{\mathbf{y}}+\mathbf{e}_{\mathbf{x}}\right)\left(\mathbf{u}^{\star}\right) \\
& \left(\epsilon^{-1} \operatorname{div}_{\mathbf{y}}+\operatorname{div}_{\mathbf{x}}\right)\left(\sigma^{\star}\right)=-\rho \omega^{2} \mathbf{u}^{\star}
\end{aligned}
$$

We consider situations where the boundary layer plays an effective role. In other words, the surface forces $\mathbf{t}$, the macro-field stress $\sigma$, and the boundary layer stress $\sigma^{\star}$, should be of the same order of magnitude. This implies that the expansions of the surface forces and of the BL stresses take the form:

$$
\mathbf{t}\left(\mathbf{x}_{\alpha}, \mathbf{y}\right)=\sum_{\mathbf{i}=\mathbf{0}}^{\infty} \epsilon^{\mathrm{i}} \mathbf{t}^{\mathrm{i}}\left(\mathbf{x}_{\alpha}, \mathbf{y}\right), \quad \sigma^{\star}\left(\mathbf{x}_{\alpha}, \mathbf{y}\right)=\sum_{\mathbf{i}=\mathbf{0}}^{\infty} \epsilon^{\mathrm{i}} \sigma^{\star \mathbf{i}}\left(\mathbf{x}_{\alpha}, \mathbf{y}\right)
$$

and consequently the BL displacement expansion begins at the order $\epsilon^{1}$, (see the definition (4) of $\sigma^{\star}$ )

$$
\mathbf{u}^{\star}\left(\mathbf{x}_{\alpha}, \mathbf{y}\right)=\sum_{\mathbf{i}=\mathbf{1}}^{\infty} \epsilon^{\mathbf{i}} \mathbf{u}^{\star \mathbf{i}}\left(\mathbf{x}_{\alpha}, \mathbf{y}\right)
$$

All the terms of the expansions in the boundary layer fulfill the condition of $\Sigma_{0}$-periodicity according to the $y_{\alpha}$ variables. Moreover, by principle:

- on $\Gamma\left(y_{3}=0\right)$, the total stress field balances the surface forces:

$$
-\left(\sigma+\sigma^{\star}\right) \cdot \mathbf{e}_{3}=\mathbf{t} \quad \text { when } y_{3}=0
$$

- far from $\Gamma$ (from the local scale point of view), i.e. when $y_{3} \rightarrow \infty$, the total field should only present variations according to the macro-scale. Thus the small scale variations of the BL field vanish, which leads to the condition:

$$
\nabla_{\mathbf{y}} \mathbf{u}^{\star} \rightarrow 0 \quad \text { when } y_{3} \rightarrow \infty
$$

\subsubsection{Other notations for macro-variables}

Later on, the following notations will be used in the macro-descriptions (no summation on repeated indices i):

$$
\begin{aligned}
& \mathbf{U}^{\mathrm{i}}(\mathbf{x})=\epsilon^{\mathrm{i}} \mathbf{u}^{\mathrm{i}}(\mathbf{x}), \quad \mathbf{U}^{\star 1}\left(\mathbf{x}_{\alpha}\right)=\epsilon \tilde{\mathbf{u}}^{\star 1}\left(\mathbf{x}_{\alpha}\right), \quad \tilde{\mathbf{u}}^{\star \mathbf{1}}=\frac{\mathbf{1}}{\left|\Sigma_{\mathbf{0}}\right|} \int_{\Sigma_{\mathbf{0}}} \mathbf{u}^{\star \mathbf{1}} \mathrm{d} s \\
& \mathbf{T}^{\mathrm{i}}\left(\mathbf{x}_{\alpha}\right)=\epsilon^{\mathrm{i}} \tilde{\mathbf{t}}^{\mathrm{i}}\left(\mathbf{x}_{\alpha}\right), \quad \widetilde{\mathbf{t}}^{\mathrm{i}}\left(\mathbf{x}_{\alpha}\right)=\frac{\mathbf{1}}{\left|\Sigma_{\mathbf{0}}\right|} \int_{\Sigma_{\mathbf{0}}} \mathbf{t}^{\mathrm{i}}\left(\mathbf{x}_{\alpha}, \mathbf{y}\right) \mathrm{d} s
\end{aligned}
$$

\subsection{Homogenisation procedure}

The formal developments follow the homogenisation procedure [12], the convergence aspect will not be treated in the present paper. The terms of different orders appearing in the balance equations are separated and the associated problems are solved successively. At each order, it is shown that the missed boundary condition for the macro-field can be derived from the analysis of the boundary layer problem. Thus, the 
macro-field is determined, enabling the boundary layer problem at the next order to be solved, and so on... In this study the resolution is performed to the second order.

\subsubsection{Remarks on $\epsilon$}

In the mathematical process $\epsilon$ is an infinitesimal quantity that allows to solve separately the problems at different orders. This ideal situation is never reached in practical applications because both micro and macro-scale are finite. Then the actual physical value of $\epsilon$ is a small but finite quantity, and the macro-descriptions at a given order $\epsilon^{i}$ should not be considered as exact but simply correct to the precision $\epsilon^{i+1}$. Thus the closer $\epsilon$ is to 1 , the larger the higher terms may influence the description. This explains the interest of investigating higher orders, specially for frequencies approaching the high bound of the low frequency range (i.e. $\lambda / 2 \pi \rightarrow l)$, when scattering effects begin to take place [5].

\section{Local and non-local macro-boundary condition}

In this section, the macroscopic boundary conditions equivalent to locally periodic surface forces are established. As the surface forces are intended to represent the effect of a resonant surface subjected to harmonic waves, they are assumed to oscillate in time at the frequency $f=\omega / 2 \pi$. However, the procedure and the results also apply for prescribed static force distributions, provided that the scale separation is satisfied.

\subsection{Macro-description at the leading order}

The zero order description is fully determined by the field $\mathbf{u}^{\mathbf{0}}(\mathbf{x}) \equiv \mathbf{U}^{\mathbf{0}}(\mathbf{x})$ governed by the elastodynamic Eq. (3) at the order $\epsilon^{0}$ and the boundary condition $(13,14)$ :

$$
\begin{aligned}
& \operatorname{div}_{\mathbf{x}}\left[\mathbf{C}: \mathbf{e}_{\mathbf{x}}\left(\mathbf{U}^{\mathbf{0}}\right)\right]=-\rho \omega^{2} \mathbf{U}^{\mathbf{0}} \\
& -\left[\mathbf{C}: \mathbf{e}_{\mathbf{x}}\left(\mathbf{U}^{\mathbf{0}}\right)\right] \cdot \mathbf{e}_{3}=\mathbf{T}^{0} \quad \text { on } x_{3}=0 \\
& \mathbf{T}^{0}=-\left|\Sigma_{0}\right|^{-1} \int_{\Sigma_{0}} \mathbf{t}^{\mathbf{0}} \mathrm{d} s
\end{aligned}
$$

The boundary condition is derived from a compatibility condition as demonstrated hereafter. Consider the first problem in the boundary layer defined by Eq. (5) at the order $\epsilon^{-1}$ and (8), (9) at the order $\epsilon^{0}$ :

$$
\begin{aligned}
& \operatorname{div}_{\mathbf{y}}\left[\mathbf{C}: \mathbf{e}_{\mathbf{y}}\left(\mathbf{u}^{\star \mathbf{1}}\right)\right]=\mathbf{0} \\
& -\mathbf{C}:\left[\mathbf{e}_{\mathbf{y}}\left(\mathbf{u}^{\star \mathbf{1}}\right)+\mathbf{e}_{\mathbf{x}}\left(\mathbf{u}^{\mathbf{0}}\right)\right] \cdot \mathbf{e}_{3}=\mathbf{t}^{\mathbf{0}} \text { on } y_{3}=0 \\
& \nabla_{\mathbf{y}} \mathbf{u}^{\star \mathbf{1}} \rightarrow \mathbf{0} \quad \text { when } y_{3} \rightarrow \infty \\
& \mathbf{u}^{\star \mathbf{1}} \text { and } \mathbf{t}^{\mathbf{0}} \quad \sum_{0} \text {-periodic in } y_{\alpha}
\end{aligned}
$$

This local problem, where $\mathbf{x}_{\alpha}$ is a parameter, is defined on the representative volume of the boundary layer, i.e. the semi-infinite cylindrical volume based on the period $\Sigma_{0} ; \Omega=\Sigma_{0} \times\left[y_{3} \leqslant 0\right]$, Fig. 2 . In the following, $\Sigma_{0}$ and $\Sigma_{\infty}$ respectively stand for the surface on $y_{3}=0$ and $y_{3} \rightarrow \infty$.

A compatibility condition must be satisfied to ensure the existence of a solution. This condition is obtained by expressing the global equilibrium of $\Omega$, taking into account the $\Sigma_{0}$-periodicity, the vanishing gradient condition and the condition on $\Sigma_{0}$. Integrating equation (15) on $\Omega$ and using the divergence theorem gives

$$
\int_{\partial \Omega}\left[\mathbf{C}: \mathbf{e}_{\mathbf{y}}\left(\mathbf{u}^{\star \mathbf{1}}\right)\right] \cdot \mathbf{n} \mathrm{d} s=0
$$

The $\Sigma_{0}$-periodicity and vanishing gradient at infinity, yield:

$$
-\int_{\Sigma_{0}}\left[\mathbf{C}: \mathbf{e}_{\mathbf{y}}\left(\mathbf{u}^{\star \mathbf{1}}\right)\right] \cdot \mathbf{e}_{\mathbf{3}} \mathrm{d} s=\mathbf{0}
$$




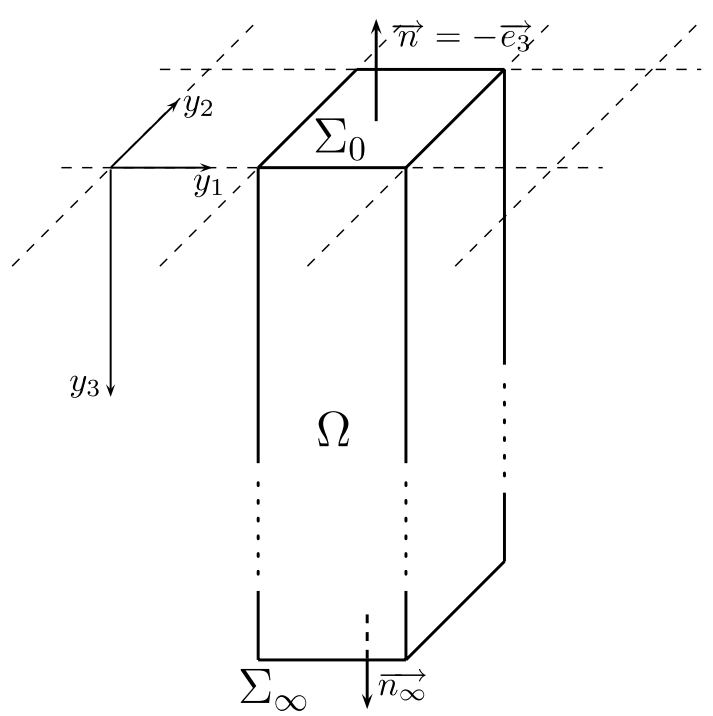

Fig. 2. The periodic volume $\Omega$ used for the definition of the boundary layer local problem.

so that, from the boundary condition on $y_{3}=0$ :

$$
\int_{\Sigma_{0}} \mathbf{t}^{\mathbf{0}} \mathrm{d} s=-\int_{\Sigma_{0}}\left[\mathbf{C}: \mathbf{e}_{\mathbf{x}}\left(\mathbf{u}^{\mathbf{0}}\right)\right] \cdot \mathbf{e}_{\mathbf{3}} \mathrm{d} s=-\left|\Sigma_{\mathbf{0}}\right|\left[\mathbf{C}: \mathbf{e}_{\mathbf{x}}\left(\mathbf{u}^{\mathbf{0}}\right)\right] \cdot \mathbf{e}_{\mathbf{3}}
$$

that supplies the boundary condition for the macro-field at the zero order. It is worth noting that this condition does not require the knowledge of the medium behaviour. At the leading order, the homogenised boundary condition is simply given by the mean value of the surface forces: the exact distribution has no influence. As a consequence, forces distribution of zero mean value (for instance a distribution of momentum) lead to the usual free surface condition. Hence, at the leading order, the effect of a periodic distribution of momentum is confined near the boundary and no macro-field is radiated.

The resolution of set (12)-(14) provides a zero order approximation sufficiently good when the scale separation is large, i.e. when $l \ll \lambda / 2 \pi$. When considering a less marked scale separation, the description can be improved by taking into account the higher order terms. For this purpose, the local problem must be solved before the first corrector of the macro-field is determined.

\subsection{First order correctors}

The first correctors consist of the BL field $\mathbf{U}^{\star \mathbf{1}}=\epsilon \mathbf{u}^{\star \mathbf{1}}$ and the macro-field $\mathbf{U}^{\mathbf{1}}=\epsilon \mathbf{u}^{\mathbf{1}}$. The results established in this section may be summarised concisely as follows. The BL field takes the form:

$$
\mathbf{U}^{\star 1}\left(\mathbf{x}_{\alpha}, \mathbf{y}\right)=\mathbf{A}(\mathbf{y}) \cdot \mathbf{T}^{\mathbf{0}}\left(\mathbf{x}_{\alpha}\right)
$$

and the first corrector of the macro-field is governed by (3) at the order $\epsilon$ and the boundary condition $(22,23)$ derived by homogenisation:

$$
\begin{aligned}
& \operatorname{div}_{\mathbf{x}}\left[\mathbf{C}: \mathbf{e}_{\mathbf{x}}\left(\mathbf{U}^{\mathbf{1}}\right)\right]=-\rho \omega^{2} \mathbf{U}^{\mathbf{1}} \\
& -\left[\mathbf{C}: \mathbf{e}_{\mathbf{x}}\left(\mathbf{U}^{1}\right)\right] \cdot \mathbf{e}_{3}=\mathbf{T}^{\mathbf{1}}-\mathbf{D} \cdot \nabla_{\mathbf{x}_{\alpha}} \mathbf{T}^{0} \quad \text { on } x_{3}=0 \\
& \mathbf{T}^{1}=-\left|\Sigma_{0}\right|^{-1} \int_{\Sigma_{0}} \mathbf{t}^{\mathbf{1}} \mathrm{d} s
\end{aligned}
$$

The features of the tensors $\mathbf{A}$ and $\mathbf{D}$ are detailed later. It is remarkable that the boundary condition (22) involves, in addition to the average force of first order, a term related to the gradient of the average force of zero order. This unusual term can be considered as a non-local contribution in the boundary condition. Obviously, when the mean surface force is uniform at the macro-scale the non-local term vanishes. This result is analogous with the higher gradient continuum theories of heterogeneous media. 


\subsubsection{Features of the boundary layer field $\boldsymbol{u}^{\boldsymbol{} \star}$}

Normalise the imposed force distribution $\mathbf{t}^{\mathbf{0}}$ on $\Sigma_{0}$ by its mean value $\mathbf{T}^{\mathbf{0}} \neq \mathbf{0}$ and introduce the functions $\tau_{i}\left(y_{\alpha}\right)=t_{i}^{0} / T_{i}^{0}$ (no summation on $i, \mathrm{O}\left(\tau_{i}\right)=1$ ) specific for the considered problem. These $\Sigma_{0}$-periodic functions may have complex values if the harmonic surface forces are out of phase.

Using the compatibility condition $(19,16)$ becomes:

$$
-\left[\mathbf{C}: \mathbf{e}_{\mathbf{y}}\left(\mathbf{u}^{\star 1}\right)\right] \mathbf{e}_{\mathbf{3}}=\mathbf{T}^{\mathbf{0}}-\mathbf{t}^{\mathbf{0}}=(\mathbf{I}-\tau) \mathbf{T}^{\mathbf{0}}
$$

where $\mathbf{I}$ is the unit matrix and $\tau$ the diagonal matrix, $\tau_{i j}=\tau_{i} \delta_{i j}$ The set (15), (17), (18), (24) constitutes a linear elastic problem concerning the volume $\Omega$ loaded on the top surface $\Sigma_{0}$ by a forcing term of zero mean value. The solution is determined up to a rigid body translation, since the $2 \mathrm{D}-\Sigma_{0}$-periodicity avoid rotations in any 3D directions. Then, introducing the additional condition:

$$
\int_{\Sigma_{\infty}} \mathbf{u}^{\star 1} \mathrm{~d} \mathbf{s} \rightarrow \mathbf{0}
$$

the solution is uniquely determined. Denoting by $\mathbf{a}^{\mathbf{i}}$ the three particular solutions corresponding to unit mean force $\mathbf{T}^{\mathbf{0}}$ in each directions $\left(T_{j}^{0}=\delta_{i j}\right)$, the boundary layer field of vanishing average value over $\Sigma_{\infty}$, reads

$$
\mathbf{u}^{\star \mathbf{1}}\left(\mathbf{x}_{\alpha}, \mathbf{y}\right)=\mathbf{a}^{\mathbf{i}}(\mathbf{y}) \mathbf{T}_{\mathbf{i}}^{\mathbf{0}}\left(\mathbf{x}_{\alpha}\right) \text { i.e. on matrix form } \mathbf{u}^{\star \mathbf{1}}=\mathbf{a} \cdot \mathbf{T}^{\mathbf{0}}
$$

where, as $\tau$, the matrix a of components $a_{j}^{i}$ takes real (respectively complex) values when the surface forces are all in phase (respectively out of phase). Any pure translation field $\mathbf{u}\left(\mathbf{x}_{\alpha}\right)$ could be added to $\mathbf{u}^{\star \mathbf{1}}$. However such a field ú may be integrated in the first order macro-field $\mathbf{u}^{1}$, then it is set equal to zero without loss of generality.

The particular solutions $\mathbf{a}^{i}$ depend on the geometry of the surface force distribution $\tau_{i}$ on $\Sigma_{0}$ (obviously, an uniform distribution gives $\mathbf{a}^{i}=0$ ). The solutions $\mathbf{a}^{i}$ can be determined numerically using the equivalent weak formulation deduced from set (15), (17), (18), (24), (25) (no summation on $i$ in the second member):

$$
-\int_{\Omega} \mathbf{e}_{\mathbf{y}}(\mathbf{w}): \mathbf{C}: \mathbf{e}_{\mathbf{y}}\left(\mathbf{a}^{\mathbf{i}}\right) \mathrm{d} \mathbf{v}=\int_{\Sigma_{\mathbf{0}}}\left(\mathbf{1}-\tau_{\mathbf{i}}\right) \mathbf{w}_{\mathbf{i}} \mathrm{d} \mathbf{s}
$$

for any continuous derivable field $\mathbf{w}$ defined in $\Omega$ and satisfying $(17,18,25)$.

This local formulation proves that the order of magnitude of $\mathbf{a}^{i}$ is $\mathrm{O}\left(l_{y} / C\right)$, where $l_{y}$ is the period dimension expressed with $\mathbf{y}$ variables. The consistency (in terms of length units) imposes the same system of unit (associated to variables) in a given set of equation. The macro-description being expressed using variables $\mathbf{x}$, all the quantities have to be expressed in this system. Thus, the true intrinsic tensor-independent of the scale ratiorelated to the physical field $\epsilon \mathbf{u}^{\star \mathbf{1}}$ is $\mathbf{A}^{i}=\epsilon \mathbf{a}^{i}=\mathrm{O}(l / C), l$ being the dimension of period expressed in the system of variables $\mathbf{x}$.

Keeping in mind that the solution is established in harmonic regime, it is noticeable that no inertial terms appear in the set defining $\mathbf{u}^{\star 1}$. To be consistent with the physics of waves, this implies that the thickness of the boundary layer is of one order smaller than the wavelength, i.e. of the order of the characteristic size of $\Sigma_{0}$.

On the basis of conical stress diffusion, an approximated BL field appropriate to simple tooth-like stress distribution is proposed in Appendix 2.

\subsubsection{Macro-boundary condition at the order one}

The next problem in the boundary layer is governed by (5) at the order $\epsilon^{0}$ and (8), (9) at the order $\epsilon$ :

$$
\begin{aligned}
& \operatorname{div}_{\mathbf{y}}\left(\sigma^{\star \mathbf{1}}\right)+\mathbf{d i v}_{\mathbf{x}}\left(\sigma^{\star \mathbf{0}}\right)=\mathbf{0} \\
& -\left(\sigma^{\mathbf{1}}+\sigma^{\star \mathbf{1}}\right) \cdot \mathbf{e}_{\mathbf{3}}=\mathbf{t}^{\mathbf{1}} \text { for } y_{3}=0 \\
& \nabla_{y} \mathbf{u}^{\star 2} \rightarrow \mathbf{0} ; \quad \sigma^{\star 1} \rightarrow \mathbf{0} \quad \text { when } y_{3} \rightarrow \infty \\
& \mathbf{u}^{\star 2} \text { and } \mathbf{t}^{1} \quad \Sigma_{0} \text {-periodic in } y_{\alpha}
\end{aligned}
$$

As above, the compatibility condition of this second local problem is derived by integrating the balance Eq. (27) on the volume $\Omega$. Let us examine separately the contributions of the stress fields $\sigma^{\star 1}$ and $\sigma^{\star 0}$. 
As for the integral related to $\sigma^{\star 1}$, the divergence theorem and the $\Sigma_{0}$-periodicity yields:

$$
\int_{\Omega} \operatorname{div}_{\mathbf{y}}\left(\sigma^{\star 1}\right) \mathrm{d} v=\int_{\partial \Omega} \sigma^{\star 1} \cdot \mathbf{n} \mathrm{d} s=\mathbf{0}-\int_{\Sigma_{0}} \sigma^{\star 1} \cdot \mathbf{e}_{3} \mathrm{~d} s+\int_{\Sigma_{\infty}} \sigma^{\star 1} \cdot \mathbf{e}_{3} \mathrm{~d} s
$$

The condition (28) on $\Sigma_{0}$ gives

$$
-\int_{\Sigma_{0}} \sigma^{\star 1} \cdot \mathbf{e}_{3} \mathrm{~d} s=\int_{\Sigma_{0}} \sigma^{1} \cdot \mathbf{e}_{3} \mathrm{~d} s+\int_{\Sigma_{0}} \mathbf{t}^{1} \mathrm{~d} s=\left|\Sigma_{\mathbf{0}}\right|\left(\sigma^{1} \cdot \mathbf{e}_{3}+\tilde{\mathbf{t}}^{1}\right)
$$

and from the expressions of $\sigma^{\star 1}$ (4) and of the BL field (26):

$$
\int_{\Sigma_{\infty}} \sigma^{\star 1} \cdot \mathbf{e}_{3} \mathrm{~d} s=\int_{\Sigma_{\infty}}\left[\mathbf{C}:\left(\mathbf{e}_{\mathbf{y}}\left(\mathbf{u}^{\star 2}\right)+\mathbf{e}_{\mathbf{x}}\left(\mathbf{u}^{\star 1}\right)\right)\right] \cdot \mathbf{e}_{3} \mathrm{~d} s=\mathbf{C}:\left[\int_{\Sigma_{\infty}} \mathbf{e}_{\mathbf{y}}\left(\mathbf{u}^{\star 2}\right) \mathrm{d} s+\mathbf{e}_{\mathbf{x}}\left(\mathbf{T}^{\mathbf{0}} \cdot \int_{\Sigma_{\infty}} \mathbf{a} \mathrm{d} s\right)\right] \cdot \mathbf{e}_{\mathbf{3}}=\mathbf{0}
$$

Indeed both integrals cancel out, the first because of the vanishing gradient condition (29), the second, because of the vanishing average value of the BL field (25) at infinity.

As for the integral related to $\sigma^{\star 0}$ the inversion of $x$-derivation and $y$-integration and the divergence theorem lead to

$$
\int_{\Omega} \operatorname{div}_{\mathbf{x}}\left(\sigma^{\star \mathbf{0}}\right) \mathrm{d} v=\operatorname{div}_{\mathbf{x}}\left[\mathbf{C}: \int_{\Omega} \mathbf{e}_{\mathbf{y}}\left(\mathbf{u}^{\star 1}\right) \mathrm{d} v\right]=\operatorname{div}_{\mathbf{x}}\left[\mathbf{C}: \int_{\partial \Omega} \frac{1}{2}\left(\mathbf{u}^{\star \mathbf{1}} \otimes \mathbf{n}+\mathbf{n} \otimes \mathbf{u}^{\star \mathbf{1}}\right) \mathrm{d} s\right]
$$

then, from the $\Sigma_{0}$-periodicity, the vanishing mean value (25) of the BL field at infinity and the expression (26) of $\mathbf{u}^{\star 1}$ :

$$
\int_{\Omega} \operatorname{div}_{\mathbf{x}}\left(\sigma^{\star 0}\right) \mathrm{d} v=-\mathbf{d i v}_{\mathbf{x}}\left[\mathbf{C}: \int_{\Sigma_{\mathbf{0}}} \frac{1}{2}\left(\left(\mathbf{a} \cdot \mathbf{T}^{\mathbf{0}}\right) \otimes \mathbf{e}_{\mathbf{3}}+\mathbf{e}_{\mathbf{3}} \otimes\left(\mathbf{a} \cdot \mathbf{T}^{\mathbf{0}}\right)\right) \mathrm{d} s\right]
$$

and considering the elastic tensor symmetry:

$$
\frac{1}{\left|\Sigma_{0}\right|} \int_{\Omega} \operatorname{div}_{\mathbf{x}}\left(\sigma^{\star \mathbf{0}}\right) \mathrm{d} v=-\mathbf{d i v}_{\mathbf{x}}\left[\mathbf{C}:\left(\left(\tilde{\mathbf{a}} \cdot \mathbf{T}^{\mathbf{0}}\right) \otimes \mathbf{e}_{3}\right)\right]=-\tilde{\mathbf{d}} \cdot \nabla_{\mathbf{x}_{\alpha}} \mathbf{T}^{\mathbf{0}}=-\tilde{d}_{\mathbf{j}}^{\mathrm{i} \alpha} \mathbf{T}_{\mathbf{i}, \alpha}^{\mathbf{0}} \mathbf{e}_{\mathbf{j}}
$$

where tensor $\tilde{\mathbf{a}}$ of order $2(3 \times 3)$, and tensor $\tilde{\mathbf{d}}$ of order $3(3 \times 3 \times 2)$ have the following components:

$$
\tilde{a}_{j}^{i}=\frac{1}{\left|\Sigma_{0}\right|} \int_{\Sigma_{0}} a_{j}^{i} \mathrm{~d} s, \quad \tilde{d}_{j}^{i \alpha}=\tilde{a}_{k}^{i} C_{k 3 j \alpha}
$$

Finally, multiplied by $\epsilon$ the compatibility condition provides the following boundary condition

$$
-\left[\mathbf{C}: \mathbf{e}_{\mathbf{x}}\left(\mathbf{U}^{\mathbf{1}}\right)\right] \cdot \mathbf{e}_{3}=\tilde{\mathbf{T}}^{\mathbf{1}}-\mathbf{D}^{\mathbf{1}} \cdot \nabla_{\mathbf{x}_{\alpha}} \mathbf{T}^{\mathbf{0}} \text { on } \mathbf{x}_{\mathbf{3}}=\mathbf{0}
$$

The tensor $\tilde{\mathbf{d}}=\mathrm{O}\left(l_{y}\right)$ mixes the elastic properties of the medium and the features of the surface force distribution. In the system of macro-variables $x, \mathbf{D}^{1}=\epsilon \tilde{\mathbf{d}}=\mathrm{O}(l)$, and $\tilde{\mathbf{A}}=\epsilon \tilde{\mathbf{a}}=\mathrm{O}(l / C)$. For an isotropic medium of the Lame coefficients $\lambda, \mu$, the non-zero components of $\mathbf{D}^{1}$ are

$$
D_{\alpha}^{1 i \alpha}=\lambda \tilde{A}_{3}^{i}, \quad D_{3}^{1 i \alpha}=\mu \tilde{A}_{\alpha}^{i}
$$

If further $\tilde{\mathbf{A}}$ is diagonal $\left(\tilde{A}_{j}^{i}=\tilde{A}_{j} \delta_{j}^{i}\right)$ :

$$
\mathbf{D}^{1} \cdot \nabla_{\mathbf{x}_{\alpha}} \mathbf{T}^{\mathbf{0}}=\lambda \tilde{A}_{3}\left(T_{3,1}^{0} \mathbf{e}_{1}+T_{3,2}^{0} \mathbf{e}_{2}\right)+\mu\left(\tilde{A}_{1} T_{1,1}^{0}+\tilde{A}_{2} T_{2,2}^{0}\right) \mathbf{e}_{3}
$$

\subsubsection{Remark}

In the case - not developed here - where mean surface forces cancel out, the normalisation should be based on momentum. Except this point, the above results formally apply in a similar way replacing mean force by momentum. Thus, even in this case, the free surface condition must be corrected at the order one, meaning that a first order macro-field is radiated by a quasi-periodic distribution of momentum. 


\subsection{Following orders}

The descriptions including the following orders could be obtained in a similar way. The same elastodynamic balance equation holds at each order. The general form of the macro-boundary condition is

$$
-\left[\mathbf{C}: \mathbf{e}_{\mathbf{x}}\left(\mathbf{U}^{\mathbf{n}}\right)\right] \cdot \mathbf{e}_{\mathbf{3}}=\mathbf{T}^{\mathbf{n}}-\sum_{\mathbf{i}=\mathbf{0}}^{\mathbf{n}-\mathbf{1}} \mathbf{D}^{\mathbf{n}-\mathbf{i}} \cdot \nabla_{\mathbf{x} \alpha}^{\mathbf{n}-\mathbf{i}} \mathbf{T}^{\mathbf{i}} \quad \text { on } x_{3}=\mathbf{0}
$$

The higher order tensors $\mathbf{D}^{i}$ of order $2+i,(3 \times 3 \times 2 \times \cdots \times 2)$ are determined by the BL fields of order $i+1$, and $\mathrm{O}\left(D^{i}\right)=l^{i}$. The BL fields at each order are governed by similar elastostatic problems except for the forcing terms. The latter are associated to the preceding BL fields and to the successive gradients of the macro-field at the previous orders. Obviously, the non-local terms disappear for mean forces macro-homogenous at any order.

\section{Macro-description of a resonant surface}

We come now to the case of a periodic distribution of linear oscillators. To avoid unnecessary complications, a single oscillator, having a rigid base of surface $S=s . \Sigma_{0}, s<1$, is located on the period $\Sigma_{0}$ (Fig. 3). This situation partly differs from the previous case because:

- the surface forces result from the oscillator behaviour,

- the forces are transmitted through the rigid base of the oscillators.

Nevertheless, it can be shown (see Appendix 1) that formally, the results established in the previous section hold in this slightly different context.

\subsection{Oscillator impedance and mean surface force distribution}

The oscillator is assumed to behave as a single degree of freedom system in each direction $\mathbf{e}_{j}$. Denoting respectively by $k_{j}$ and $c_{j}$ the stiffness and the viscous damping coefficient in the direction $j$ and the mass by $m$, the resonant frequency $f_{j}=\omega_{j} / 2 \pi$ and the damping ratio are calculated as

$$
\omega_{j}=2 \pi f_{j}=\sqrt{k_{j} / m} \quad \xi_{j}=c_{j} / 2 \sqrt{k_{j} m}
$$

As usual, a weak damping (i.e. $\xi_{j}$ of the order of $10^{-2}$ or less) will be considered.

The harmonic behaviour at the forced frequency $f=\omega / 2 \pi$, can be derived similarly for each direction $\mathbf{e}_{j}$. Denoting $\mathbf{U}_{m}$ the translation motion of the mass and $\mathbf{U}_{s}$ the base motion, the non-zero component of the resultant force, $R_{j}$, is given by (no summation on $j$ ):

$$
-\left(k_{j}-\mathrm{i} \omega c_{j}\right)\left(U_{m j}-U_{S j}\right)=R_{j}
$$

and the balance equation of the mass reads

$$
-\left(k_{j}-\mathrm{i} \omega c_{j}\right)\left(U_{m j}-U_{S j}\right)=-m \omega^{2} U_{m j}
$$

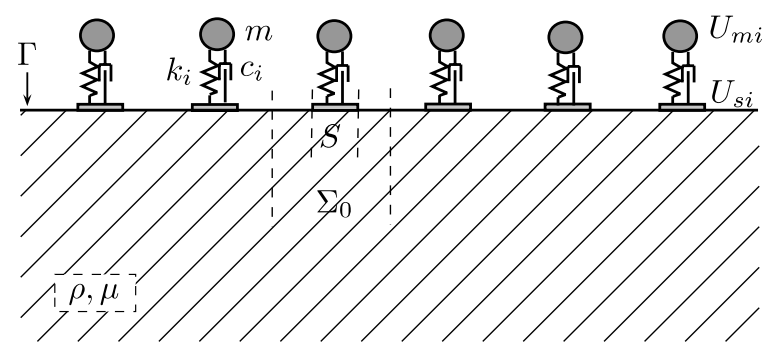

Fig. 3. Half-space loaded by a periodic distribution of $3 \mathrm{D}$ oscillators. 
Then, eliminating the mass motion (no summation on $j$ ):

$$
R_{j}=F_{j}(\omega) U_{S j}, \quad F_{j}(\omega)=m \omega^{2} \frac{\omega_{j}^{2}+2 \mathrm{i} \xi_{j} \omega \omega_{j}}{\omega_{j}^{2}+2 \mathrm{i} \xi_{j} \omega \omega_{j}-\omega^{2}}
$$

The transfer function, $F_{j}(\omega)$, shows that at low frequency (compared to the natural frequency $\omega_{j}$ ) the oscillator acts as a mass $\left(F_{j} \approx m \omega^{2}\right)$, and at high frequency as a spring-damper system $\left(F_{j} \approx k_{j}-\mathrm{i} \omega c_{j}\right)$. In the vicinity of the natural frequency, the resonant amplification leads to much larger values $\left(F_{j} \approx k_{j} / 2 \mathrm{i} \xi_{j}\right)$.

The diagonal impedance matrix $\mathbf{Z}$ that relates the resultant force $\mathbf{R}$ to the oscillator base translation $\mathbf{U}_{\mathbf{S}}$ is derived from the transfer diagonal matrix $\left(\mathbf{F}, F_{i j}=\delta_{i j} F_{j}\right)$ :

$$
\mathbf{R}=\mathrm{i} \omega \mathbf{Z} \cdot \mathbf{U}_{\mathbf{S}}, \quad \text { where } \mathbf{Z}(\omega)=\frac{\mathbf{F}(\omega)}{\mathrm{i} \omega}
$$

Outside the oscillator base, the surface remains free, i.e. $\mathbf{t}=0$. Inside $S$, the exact surface force distribution is unknown but $\mathbf{t}$ balances the resultant force $\mathbf{R}$ due to the oscillator:

$$
\mathbf{T}=\left|\Sigma_{0}\right|^{-1} \int_{\Sigma_{0}} \mathbf{t} \mathrm{d} s=\left|\Sigma_{0}\right|^{-1} \int_{S} \mathbf{t} \mathrm{d} s=\left|\Sigma_{0}\right|^{-1} \mathbf{R}=\left|\Sigma_{0}\right|^{-1} \mathrm{i} \omega \mathbf{Z} \cdot \mathbf{U}_{\mathbf{S}}
$$

Denoting

$$
\mathbf{Z}^{0}=\mathbf{Z} /\left|\Sigma_{0}\right|
$$

then

$$
\mathbf{T}=\mathrm{i} \omega \mathbf{Z}^{0} \cdot \mathbf{U}_{\mathbf{S}}
$$

From the results of the previous section, the base translation is expanded in the form:

$$
\mathbf{U}_{\mathbf{S}}=|S|^{-1} \int_{S} \mathbf{U d} s=\mathbf{U}^{0}+\left(\mathbf{U}^{1}+|S|^{-1} \int_{S} \mathbf{U}^{\star 1} \mathrm{~d} s\right)+\cdots
$$

\subsection{Equivalent surface impedance at the leading order}

We are now in a position to identify the boundary condition equivalent to a resonant surface at the leading order. Eqs. (35), (36) at zero order yield

$$
\mathbf{T}^{\mathbf{0}}=\mathrm{i} \omega \mathbf{Z}^{\mathbf{0}} \cdot \mathbf{U}^{\mathbf{0}}\left(x_{\alpha}, x_{3}=0\right)
$$

and the zero order boundary condition (13) takes the form

$$
-\left[\mathbf{C}: \mathbf{e}_{\mathbf{x}}\left(\mathbf{U}^{\mathbf{0}}\right)\right] \cdot \mathbf{e}_{\mathbf{3}}=\mathrm{i} \omega \mathbf{Z}^{\mathbf{0}} \cdot \mathbf{U}^{\mathbf{0}} \quad \text { on } x_{3}=\mathbf{0}
$$

$\mathbf{Z}^{0}$ is the mean surface impedance matrix equivalent to the oscillators. Such result is used in the "fuzzy structure" approach [14,15] and in electromagnetics [9]. Situations with a finite number of oscillators $I$, of different impedances $\mathbf{Z}_{I}$, orientated in various directions, would lead to: $\mathbf{Z}^{0}=\left(\sum \mathbf{Z}_{I}\right) /\left|\Sigma_{0}\right|$.

\subsubsection{Isotropic and anisotropic resonant surface}

As mentioned above, the impedance strongly depends on the frequency and the effect of oscillator dominates at its eigen frequencies. Then, focusing on the two directions of the interface $\Gamma$, the mean impedance matrix may be:

- either isotropic, i.e. $Z_{1}(\omega)=Z_{2}(\omega)$, this will be the case if the oscillator presents the same characteristics in both directions,

- or anisotropic, with an anisotropy ratio varying with the frequency if the two natural frequencies $\left(\omega_{1}, \omega_{2}\right)$ are different. For instance, if $\omega_{2}=2 \omega_{1}$, according to the transfer functions (32), $\left|Z_{2}\left(\omega_{1}\right)\right| /\left|Z_{1}\left(\omega_{1}\right)\right|=$ $\left|Z_{1}\left(\omega_{2}\right)\right| /\left|Z_{2}\left(\omega_{2}\right)\right|=\xi / 3$, i.e. $\left|Z_{2}\right| \ll\left|Z_{1}\right|$ around $\omega_{1}$ and inversely, $\left|Z_{1}\right| \ll\left|Z_{2}\right|$ around $\omega_{2}$. More generally 
if the oscillator is perfectly rigid in one direction, then its impedance in this direction is negligible compared to the impedance in the other directions, at least around resonant frequencies.

\subsection{Local and non-local impedance correctors}

The next order boundary condition (31) needs to evaluate the local and non-local term to be balanced. From the expression (37) of $\mathbf{T}^{0}$ and of the boundary layer field (26) the first order base motion becomes (B stand for the base translations due to the BL field, see Appendices 1 and 2 for estimates):

$$
\mathbf{U}_{S}^{1}=\mathbf{U}^{1}+|S|^{-1} \int_{S} \mathbf{U}^{\star 1} \mathrm{~d} s=\mathbf{U}^{1}+\mathbf{B} \cdot \mathbf{T}^{0}=\mathbf{U}^{1}+\mathbf{B} \cdot\left(\mathrm{i} \omega \mathbf{Z}^{0}\right) \cdot \mathbf{U}^{0}
$$

Now, from the oscillator behaviour

$$
\mathbf{T}^{1}=\mathrm{i} \omega \mathbf{Z}^{0} \cdot \mathbf{U}_{\mathbf{S}}^{\mathbf{1}}=\mathrm{i} \omega \mathbf{Z}^{0} \mathbf{U}^{1}+\left(\mathrm{i} \omega \mathbf{Z}^{0}\right) \cdot \mathbf{B} \cdot\left(\mathrm{i} \omega \mathbf{Z}^{0}\right) \mathbf{U}^{0}
$$

The non-local term is calculated as

$$
\mathbf{D}^{\mathbf{1}} \cdot \nabla_{\mathbf{x} \alpha} \mathbf{T}^{\mathbf{0}}=\mathrm{i} \omega \mathbf{D}^{\mathbf{1}} \cdot \mathbf{Z}^{\mathbf{0}} \cdot \nabla_{\mathbf{x} \alpha} \mathbf{U}^{\mathbf{0}}=\mathrm{i} \omega \mathbf{Y}^{\mathbf{1}} \cdot \nabla_{\mathbf{x}_{\alpha}} \mathbf{U}^{\mathbf{0}}
$$

and the boundary condition respected by the first corrector of the macro-field takes the form

$$
-\left[\mathbf{C}: \mathbf{e}_{\mathbf{x}}\left(\mathbf{U}^{\mathbf{1}}\right)\right] \cdot \mathbf{e}_{\mathbf{3}}-\mathbf{i} \omega \mathbf{Z}^{\mathbf{0}} \cdot \mathbf{U}^{\mathbf{1}}=-\omega^{2} \mathbf{Z}^{\mathbf{1}} \cdot \mathbf{U}^{\mathbf{0}}+\mathrm{i} \omega \mathbf{Y}^{\mathbf{1}} \cdot \nabla_{\mathbf{x}_{\alpha}} \mathbf{U}^{\mathbf{0}} \quad \text { on } x_{\mathbf{3}}=\mathbf{0}
$$

where

$$
\mathbf{Z}^{1}=\mathbf{Z}^{0} \cdot \mathbf{B} \cdot \mathbf{Z}^{0}, \quad \mathbf{Y}^{1}=\mathbf{D}^{\mathbf{1}} \cdot \mathbf{Z}^{\mathbf{0}}
$$

The matrix $\mathbf{Z}^{1}$ constitutes the first corrector of the surface impedance and $\mathbf{Y}^{1}$ stands for the non-local impedance matrix of third order $(3 \times 3 \times 2)$.

\subsubsection{Tensor estimates}

The evaluation of correctors requires the knowledge of the several tensors associated to the BL field. In the following, we systematically use the estimated BL field established in Appendix 2 for an isotropic medium of rigidity $\mu$ and Poisson ratio $v$, that leads to the values summarised below. $\tilde{\mathbf{A}}$ and $\mathbf{B}$ are diagonal tensors $\left(\tilde{A}_{j}^{i}=\tilde{A}_{j} \delta_{j}^{i} ; B_{j}^{i}=B_{j} \delta_{j}^{i}\right)$ of components:

$$
\begin{aligned}
& \tilde{A}_{1}=\tilde{A}_{2}=l \cdot \frac{\pi}{8} \frac{2-v}{\mu}(1-\sqrt{s}), \quad \tilde{A}_{3}=l \cdot \frac{\pi}{8} \frac{1-v}{\mu}\left[1+\frac{1-2 v}{(1-v)^{2}}\right](1-\sqrt{s}) \\
& B_{1}=B_{2}=-l \cdot \frac{\pi}{8} \frac{2-v}{\mu} \frac{(1-\sqrt{s})^{2}}{2 \sqrt{s}}, \quad B_{3}=-l \cdot \frac{\pi}{8} \frac{1-v}{\mu}\left[1-\frac{1-2 v}{(1-v)^{2}} \sqrt{s}\right] \frac{1-\sqrt{s}}{\sqrt{s}}
\end{aligned}
$$

where

$$
l=\sqrt{4 \Sigma_{0} / \pi} \text { and } s=S / \Sigma_{0}
$$

On $\Sigma_{0}$, the mean quadratic value of $\mathbf{A}$ components reads

$$
\begin{aligned}
& \underline{A}_{1}=\underline{A}_{2}=l \cdot \frac{\pi}{16} \frac{2-v}{\mu} \sqrt{(1-\sqrt{s})(5-3 \sqrt{s})} \\
& \underline{A}_{3}=l \cdot \frac{\pi}{16} \frac{1-v}{2 \mu} \sqrt{1-\sqrt{s}} \sqrt{(1-\sqrt{s})\left(1+\frac{1-2 v}{(1-v)^{2}}\right)^{2}+1+\sqrt{s}}
\end{aligned}
$$

The non-local term takes the form:

$$
\mathbf{Y}^{1} \cdot \nabla_{\mathbf{x}_{\alpha}} \mathbf{U}^{0}=\lambda \tilde{A}_{3} Z_{3}^{0}\left(U_{3,1}^{0} \mathbf{e}_{1}+U_{3,2}^{0} \mathbf{e}_{2}\right)+\mu \tilde{A}_{1}\left(Z_{1}^{0} U_{1,1}^{0}+Z_{2}^{0} U_{2,2}^{0}\right) \mathbf{e}_{3}
$$




\section{Perturbation of wave field by a resonant surface}

The perturbations of wave propagation induced by a regular distribution of oscillators is now investigated. The main features of the phenomena are illustrated from the study of plane wave refraction. The derivation will be supplied for the zero order approximation and for the correctors.

The elastic half space is assumed to be isotropic, with a shear wave (S) velocity $c_{\mathrm{S}}=\sqrt{\mu / \rho}$, and a compressional wave $(\mathrm{P})$ velocity $c_{\mathrm{P}}=\sqrt{(\lambda+2 \mu) / \rho}$. As above, a single $3 \mathrm{D}$ oscillator of surface $S=s \cdot \Sigma_{0}$ is located on $\Sigma_{0}$ and characterised by an equivalent diagonal impedance matrix: $Z_{i j}^{0}=Z_{i}^{0} \delta_{i j}$. We focus on the response to incident harmonic plane waves (here again the time dependence exp[i $[\omega t]$ is systematically omitted).

Before analysing specific cases, let us prove that the Descartes law is preserved in the presence of the resonant surface. At the leading order, the governing equations are

$$
\begin{aligned}
\operatorname{div}_{\mathbf{x}}\left[\mathbf{C}: \mathbf{e}_{\mathbf{x}}\left(\mathbf{U}^{\mathbf{0}}\right)\right] & =-\rho \omega^{2} \mathbf{U}^{\mathbf{0}} \\
-\left[\mathbf{C}: \mathbf{e}_{\mathbf{x}}\left(\mathbf{U}^{\mathbf{0}}\right)\right] \cdot \mathbf{e}_{\mathbf{3}} & =\mathrm{i} \omega \mathbf{Z}^{0} \cdot \mathbf{U}^{0} \quad \text { on } x_{3}=0
\end{aligned}
$$

Consider an incident plane wave of wave number $h,\left(h=h_{\mathrm{S}}=\omega / c_{\mathrm{S}}\right.$ for S wave, $h=h_{\mathrm{P}}=\omega / c_{\mathrm{P}}$ for $\mathrm{P}$ wave), propagating in the direction $\mathbf{d}=d_{j} \mathbf{e}_{j}(|\mathbf{d}|=1)$ :

$$
\mathbf{U}^{\text {inc }}=\mathbf{U} \exp \left[\mathrm{i} h\left(d_{\alpha} x_{\alpha}+d_{3} x_{3}\right)\right]
$$

The wave field is decomposed into the incident and the diffracted field:

$$
\mathbf{U}^{0}=\mathbf{U}^{\text {inc }}+\mathbf{U}^{\text {dif }}
$$

To fulfill the boundary condition (44) on the surface $\Gamma$ (i.e. according to variables $x_{\alpha}$ ), the incident and diffracted fields must present the same wave numbers in the plan $\Gamma$, i.e. $h \cdot d_{\alpha}$ are identical so that the Descartes law applies. For the same reason (see Section 5.1.2) this result holds at any order.

\subsection{Refraction of oblique $S H$ waves}

This subsection presents the zero and first order effect of a resonant surface on the refraction of SH plane waves of oblique incidence.

\subsubsection{Macro-description to first order}

Consider an incident $\mathrm{SH}_{1}$ wave polarised in the direction $\mathbf{e}_{1}$ coinciding with a main direction of the oscillator. Suppose further that the wave propagates obliquely in the direction $\mathbf{d}=\sin (\varphi) \mathbf{e}_{2}+\cos (\varphi) \mathbf{e}_{3}, \varphi$ being the incidence angle to the normal of the surface $\Gamma$. The incident field reads (to save notation the exponent 0 is omitted)

$$
\mathbf{U}^{\text {inc }}=U^{i} \exp \left[\mathrm{i} h_{\mathrm{S}}\left(\sin (\varphi) x_{2}+\cos (\varphi) x_{3}\right)\right] \mathbf{e}_{1}
$$

As $\mathbf{Z}^{0}$ is diagonal in the frame $\left(\mathbf{e}_{i}\right)$, the stress and motion on $\Gamma$ have the same direction of polarisation $\mathbf{e}_{1}$. Then according to the Descartes law, the diffracted field is a down warding $\mathrm{SH}_{1}$ wave

$$
\mathbf{U}^{\text {dif }}=U^{d} \exp \left[\mathrm{i} h_{\mathrm{S}}\left(\sin (\varphi) x_{2}-\cos (\varphi) x_{3}\right)\right] \mathbf{e}_{1}
$$

The boundary condition (44):

$$
-\mu \mathrm{i} h_{\mathrm{S}} \cos (\varphi)\left(U^{i}-U^{d}\right)=\mathrm{i} \omega Z_{1}\left(U^{i}+U^{d}\right)
$$

gives

$$
U^{d}=U^{i} \frac{1-\frac{P_{1}}{\cos (\varphi)}}{1+\frac{P_{1}}{\cos (\varphi)}}
$$

where

$$
P_{1}(\omega)=-\frac{\mathrm{i} \omega Z_{1}^{0}}{\mathrm{i} \mu h}=\eta_{1} \frac{\mathrm{i} \omega}{\omega_{1}} \cdot \frac{\omega_{1}^{2}+2 \mathrm{i} \xi_{1} \omega \omega_{1}}{\omega_{1}^{2}+2 \mathrm{i} \xi_{1} \omega \omega_{1}-\omega^{2}} \quad \text { and } \quad \eta_{1}=\frac{\sqrt{k_{1} m}}{\Sigma_{0} \sqrt{\mu \rho}}=\frac{\omega_{1} m}{c_{\mathrm{S}} \Sigma_{0} \rho}
$$


Except in the case of vanishing surface impedance, (46) shows that the amplitude of the diffracted field differs from that observed in the free surface situation. The function $P_{1}(\omega)$ plays the role of a frequency dependant and complex impedance ratio between the elastic half space and the resonant surface. The main features of the perturbations are detailed hereafter.

5.1.1.1. Leading parameters. The amplitude of $P_{1}(\omega)$ linearly depends on $\eta_{1}$. This dimensionless parameter that compares the properties of the oscillator and the medium provides a first indicator of the efficiency of the resonant surface.

The perturbations strongly depend on the frequency:

- at low and high frequencies (compared to the natural frequency $\omega_{1}$ ) the perturbations are negligible, as

$$
P_{1}(\omega) \rightarrow \eta_{1} \omega / \omega_{1} \quad \text { when } \omega / \omega_{1} \rightarrow 0 \quad \text { and } \quad P_{1}(\omega) \rightarrow 2 \eta_{1} \xi_{1} \quad \text { when } \omega / \omega_{1} \rightarrow \infty
$$

- in the vicinity of the natural frequency, the effect may be very significant when $\eta_{1}=\mathrm{O}(1)$ and $\xi_{1} \ll 1$, as

$$
P_{1}\left(\omega_{1}\right)=\frac{\eta_{1}}{2 \xi_{1}}\left(1+2 \mathrm{i} \xi_{1}\right) \quad \text { when } \omega=\omega_{1}
$$

5.1.1.2. Conditions for a significant effect. To obtain a significant effect at the oscillator natural frequency, it suffices that

$$
\frac{\eta_{1}}{2 \xi_{1} \cos (\varphi)} \gg \epsilon_{1} \quad \text { with } \epsilon_{1}=2 \pi l / \lambda_{1} \ll 1
$$

to satisfy the scale separation at this frequency. These two inequalities expressed in a less restrictive manner, $\eta_{1} /\left(2 \xi_{1} \cos (\varphi)\right)>\epsilon_{1}$ and $\epsilon_{1}<1$, lead to the order of magnitude of the minimum mass and maximum rigidity of the oscillators so that an influence on the macro-wave field may actually be observed. The first requirement

$$
\frac{\eta_{1}}{2 \xi_{1} \cos (\varphi)}=\frac{\omega_{1} m}{2 c_{\mathrm{S}} \Sigma_{0} \rho \xi_{1} \cos (\varphi)}>\epsilon_{1}=\frac{\omega_{1} l}{c_{\mathrm{S}}}
$$

gives

$$
m>2 \xi_{1} \cos (\varphi) M \quad \text { with } M=\rho l \Sigma_{0}=\mathrm{O}\left(\rho l^{3}\right)
$$

and the second condition:

$$
\epsilon_{1}=\frac{\omega_{1} l}{c_{\mathrm{S}}}=l \frac{\sqrt{k_{1}}}{\sqrt{m}} \frac{\sqrt{\rho}}{\sqrt{\mu}}<1 \quad \text { gives } k_{1}<\frac{m}{\rho l^{2}} \mu=\mathrm{O}\left(\frac{m}{M} \mu l\right)
$$

The inequality (48) underlines the key role of the damping and shows that even with "small" masses, a distribution of weak damped oscillators may have a significant effect near their resonance. Here "small" has to be understood in comparison with the mass $M$ of the medium contained in the "cubic" volume $l \times \Sigma_{0}$ defined from the typical size of $\Sigma_{0}$. Note from (47) that $\eta_{1}=\epsilon_{1} \mathrm{~m} / M$. Then, from practical considerations on the mass of the oscillators, the reasonable assumption $\eta_{1}<1$ is made in the following.

Furthermore, according to the homogenisation approach, the oscillator effect must be rejected to the order one for frequencies $\omega=\omega_{1}+\delta \omega$ such that $P_{1}(\omega) / \cos (\varphi)<\mathrm{O}(\epsilon)=\mathrm{O}\left(\omega l / c_{\mathrm{S}}\right)$, i.e. with the weak damping assumption:

$$
\frac{\left|P_{1}(\omega)\right|}{\cos (\varphi)} \approx \frac{\omega_{1} m}{c_{\mathrm{S}} \cos (\varphi) \Sigma_{0} \rho} \cdot \frac{\omega}{\omega_{1}} \cdot \frac{\omega_{1}^{2}}{2 \omega_{1}|\delta \omega|}<\frac{\omega l}{c_{\mathrm{S}}} \quad \text { so that } \frac{|\delta \omega|}{\omega_{1}}>\frac{m}{M \cos (\varphi)}
$$

Thus, the maximum extent of the frequency range where the resonant surface influences the leading order is given by: $\left[\omega_{1}(1 \pm m / M \cos (\varphi))\right]$. Outside of this range, the free surface condition is valid at the leading order and the perturbation decreases of one order. 
5.1.1.3. De-amplification effect. Around the resonance, the oscillator layer presents a larger impedance than the elastic medium. This situation, quite opposite to the free surface condition, leads to a significant decrease of the surface total motion $\left(U^{d} \approx-U^{i}\right.$ instead of $U^{d}=U^{i}$ for the free surface). Note that the incidence angle enhances the apparent impedance contrast, inducing in turn an effect even more marked for oblique waves.

An illustration is given in Fig. 4, where the amplitude ratio between the surface total motion and the incident motion, $\left|U^{d}+U^{i}\right| /\left|U^{i}\right|$, versus the dimensionless frequency $\omega / \omega_{1}$ are presented for several values of incidence angle. Clearly, the de-amplification effect is concentrated in the vicinity of the resonant frequency and its amplitude increases when the incident wave obliquity increases.

This finding seems to corroborate some numerical results e.g., [7], and to be in contradiction with others e.g., [16]. The reason lies in the type of oscillator's distribution considered in these works. When the distribution is sufficiently regular and extended (relatively to the wave length) the notion of representative surface element applies. Then numerical and homogenised modelling lead to similar results, at least in the scale separation frequency range. When the distribution is made of about ten oscillators all of them having different properties (e.g., Tsogka and Wirgin's case) none ERS exists and the homogeneisation approach is irrelevant. Thus, it is not surprising that numerical description in this case presents other features than in a homogenisable case. The same kind of discrepancy would be observed if the response of a group of a few (say 3-5) identical oscillators (non-homogenisable case) were compared to the response of an infinite regular distribution of these oscillators (homogenisable case).

5.1.1.4. Characteristic time of response. Investigate now the consequences in the time domain of the frequency dependence of the oscillator effect. Consider the difference $U_{d}$ of the total surface motion in presence and in absence of the oscillator layer. From (46) and (47)

$$
U_{d}=\left(U^{d}+U^{i}\right)-2 U^{i}=2 U^{i} \frac{-\frac{\eta_{1}}{\cos (\varphi)}}{1+2 \xi_{1} \frac{\eta_{1}}{\cos (\varphi)}}\left[-2 \xi \omega^{2}+\mathrm{i} \omega \omega_{1}\right] J(\omega)
$$

where

$$
J(\omega)=\frac{1}{-\omega^{2}+2 \mathrm{i} \zeta \omega \tilde{\omega}_{1}+\tilde{\omega}_{1}^{2}}, \quad \tilde{\omega}_{1}=\frac{\omega_{1}}{\sqrt{1+2 \xi_{1} \frac{\eta_{1}}{\cos (\varphi)}}}, \quad \zeta=\frac{\xi_{1}+\frac{\eta_{1}}{2 \cos (\varphi)}}{\sqrt{1+2 \xi_{1} \frac{\eta_{1}}{\cos (\varphi)}}}
$$

The time response to an incident impulsive wave, i.e. $U^{i}=\delta(t)$, is directly obtained by the inverse Fourier transform of $J(\omega)$ :

$$
\widehat{U_{d}(t)}=2 \frac{-\frac{\eta_{1}}{\cos (\varphi)}}{1+2 \xi_{1} \frac{\eta_{1}}{\cos (\varphi)}}\left[2 \xi_{1} \ddot{\widehat{J}}(t)+\omega_{1} \dot{\hat{J}}(t)\right]
$$

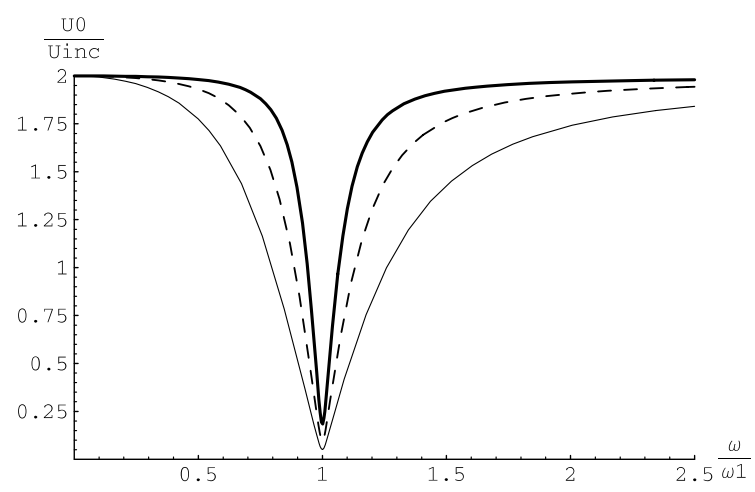

Fig. 4. Effect of a resonant surface $\left(\eta_{1} / 2 \xi_{1}=10\right)$ on the total surface motion according to $\omega / \omega_{1}$ for SH waves of different incidence angle (bold line: normal incidence, dashed line $\pi / 3$ incidence, normal line $5 \pi / 12$ incidence). The amplitude ratio between surface to incident motions would be equal to 2 for a free surface. 
where, assuming $\eta_{1} / \cos (\varphi)<1$

$$
\widehat{J}(t)=\frac{1}{\tilde{\omega}_{1} \sqrt{1-\zeta^{2}}} \sin \left[\tilde{\omega}_{1} \sqrt{1-\zeta^{2}} t\right] \exp \left[-\zeta \tilde{\omega}_{1} t\right]
$$

Therefore instead of an instantaneous response (case of a free surface), the oscillators layer induces a delayed response showing damped oscillations of pulsation $\tilde{\omega}_{1} \sqrt{1-\zeta^{2}}$ different from $\omega_{1}$ and a characteristic duration time $t_{\mathrm{c}}=2 \pi /\left(\zeta \tilde{\omega}_{1}\right) \approx 2 \pi \cos (\varphi) /\left(\eta_{1} \omega_{1}\right)$. Thus, if the resonant frequency belongs to the incident motion spectrum, it is expected that this "surface memory effect" increases the surface motion by a duration of the order of $t_{\mathrm{c}}$. Note that in presence of a top layer the surface motion may also be altered by beatings see for instance $[18,6]$.

\subsubsection{First correctors}

The macro-stresses (or displacements) on the surface are needed to evaluate the first correctors, i.e. the BL field $\mathbf{U}^{\star 1}$ and the macro-field $\mathbf{U}^{\mathbf{1}}$. From (46)

$$
\begin{aligned}
& \mathbf{U}_{\left(x_{3}=0\right)}^{0}=\mathbf{U}^{\mathrm{inc}}+\mathbf{U}^{\mathrm{dif}}=\frac{2}{1+\frac{P_{1}}{\cos (\varphi)}} \exp \left[\mathrm{i} h_{\mathrm{S}} \sin (\varphi) x_{2}\right] U^{i} \mathbf{e}_{1} \\
& \mathbf{T}_{\left(x_{3}=0\right)}^{0}=\mathrm{i} \omega \mathbf{Z}^{0} \cdot \mathbf{U}_{\left(x_{3}=0\right)}^{0}=\frac{2 \mathrm{i} \omega Z_{1}^{0}}{1+\frac{P_{1}}{\cos (\varphi)}} \exp \left[\mathrm{i} h_{\mathrm{S}} \sin (\varphi) x_{2}\right] U^{i} \mathbf{e}_{1}
\end{aligned}
$$

Begin with the BL field defined by (20). From Appendix 2, $\mathbf{A}$ is a diagonal tensor $A_{i j}=A_{i} \delta_{i j}$ so that

$$
\mathbf{U}^{\star 1}=A_{1}(\mathbf{y}) \mathbf{T}^{0}=A_{1} \frac{2 \mathrm{i} \omega Z_{1}^{0}}{1+\frac{P_{1}}{\cos (\varphi)}} \exp \left[\mathrm{i} h_{\mathrm{S}} \sin (\varphi) x_{2}\right] U^{i} \mathbf{e}_{1}
$$

Thus, on the top surface, the spatial mean quadratic deviation induced by the BL field is

$$
\underline{\mathbf{U}^{\star 1}}=\underline{A_{1}} U^{i} \frac{2 \mathrm{i} \omega Z_{1}^{0}}{1+\frac{P_{1}}{\cos (\varphi)}}=-\mu \underline{A_{1}} i \frac{\omega}{c_{\mathrm{S}}} \cos (\varphi) \frac{2 \frac{P_{1}}{\cos (\varphi)}}{1+\frac{P_{1}}{\cos (\varphi)}} U^{i} \mathbf{e}_{1}
$$

and from expression (42):

$$
\underline{\mathbf{U}^{\star 1}}=\frac{\pi(2-v)}{16} \sqrt{(1-\sqrt{s})(5-3 \sqrt{s})} \frac{\mathrm{i} \omega l}{c_{\mathrm{S}}} \cos (\varphi) \frac{2 \frac{P_{1}}{\cos (\varphi)}}{1+\frac{P_{1}}{\cos (\varphi)}} U^{i} \mathbf{e}_{1}
$$

This expression underlines the significant influence of the scale ratio $\left(\epsilon=\omega l / c_{\mathrm{S}}\right)$ conjuged with the resonance effect and shows that the BL corrector is in phase quadrature (neglecting the damping) around the natural oscillator frequency. The Fig. 5 that depicts the amplitude variations of the BL corrector with frequency,

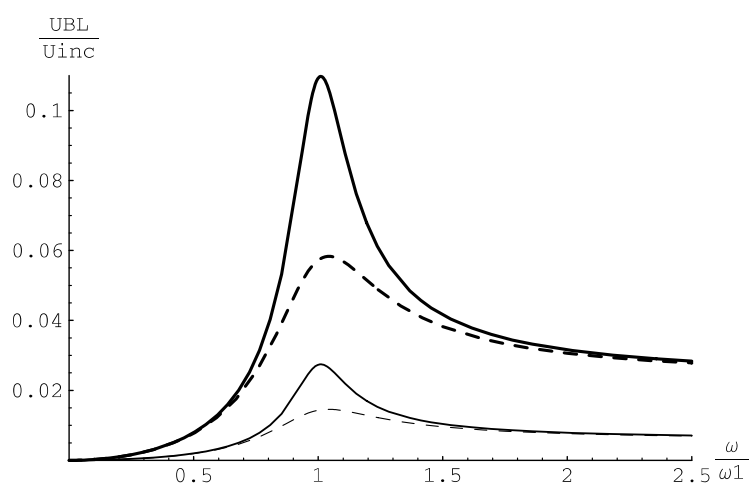

Fig. 5. Mean quadratic deviation of the BL field on the top surface according to $\omega / \omega_{1}$ for two SH incidence angles (plain line: normal incidence, dashed line $\pi / 3$ incidence $)$ and two values of $\epsilon_{1}=\omega_{1} l / c_{\mathrm{S}}\left(\epsilon_{1}=0.2\right.$ : bold lines; $\epsilon_{1}=0.05$ : normal lines $)$. Calculations performed for $\eta_{1} / 2 \xi_{1}=10, s=0.5, v=1 / 3$. 
shows the dominating effect around the oscillator eigen frequency, the significant influence of the scale ratio and of the incidence angle.

Now determine the macro-field $\mathbf{U}^{1}$ governed by the equations

$$
\begin{aligned}
& \operatorname{div}_{\mathbf{x}}\left[\mathbf{C}: \mathbf{e}_{\mathbf{x}}\left(\mathbf{U}^{1}\right)\right]+\rho \omega^{2} \mathbf{U}^{1}=0 \\
& -\left[\mathbf{C}: \mathbf{e}_{\mathbf{x}}\left(\mathbf{U}^{1}\right)\right] \cdot \mathbf{e}_{3}-\mathrm{i} \omega \mathbf{Z}^{0} \cdot \mathbf{U}^{1}=-\omega^{2} \mathbf{Z}^{1} \cdot \mathbf{U}^{0}+\mathrm{i} \omega \mathbf{Y}^{1} \cdot \nabla_{\mathrm{x}_{\alpha}} \mathbf{U}^{0} \quad \text { on } x_{3}=0
\end{aligned}
$$

In the elastic half-space with surface impedance $\mathbf{Z}^{0}, \mathbf{U}^{1}$ balances the remaining surfaces forces neglected at the leading order (second term of (56)). As these later are derived from $\mathbf{U}^{0}$, the Descartes law continues to hold for $\mathbf{U}^{1}$.

In this particular case, the non-local term cancels out:

$$
\mathbf{Y}^{1} \cdot \nabla_{x \alpha} \mathbf{U}^{0}=\mu \tilde{A}_{1} Z_{1}^{0} U_{1,1}^{0} \mathbf{e}_{3}=0
$$

and the source on the surface $\Gamma$ reduces to

$$
-\omega^{2} \mathbf{Z}^{1} \cdot \mathbf{U}^{0}=\left(\mathrm{i} \omega Z_{1}^{0}\right)^{2} B_{1} \frac{2}{1+\frac{P_{1}}{\cos (\varphi)}} \exp \left[\mathrm{i} h_{\mathrm{S}} \sin (\varphi) x_{2}\right] U^{i} \mathbf{e}_{1}
$$

The diffracted field radiated by this source is a $\mathrm{SH}_{1}$ plane wave

$$
\mathbf{U}^{1}=U^{1} \exp \left[\mathrm{i} h_{\mathrm{S}} \sin (\varphi) x_{2}-\cos (\varphi) x_{3}\right] \mathbf{e}_{1}
$$

whose amplitude is defined by the boundary condition (56):

$$
\left[\mu \mathrm{i} h_{\mathrm{S}} \cos (\varphi)-\mathrm{i} \omega Z_{1}^{0}\right] U^{1}=\left(\mathrm{i} \omega Z_{1}^{0}\right)^{2} B_{1} \frac{2}{1+\frac{P_{1}}{\cos (\varphi)}} U^{i}
$$

giving

$$
U^{1}=\mu \mathrm{i} h_{\mathrm{S}} B_{1} \cos (\varphi)\left[\frac{\frac{2 P_{1}}{\cos (\varphi)}}{1+\frac{P_{1}}{\cos (\varphi)}}\right]^{2}
$$

and from expression (41)

$$
U^{1}=-\frac{\pi}{8} \frac{2-v}{2 \sqrt{s}}(1-\sqrt{s})^{2} \frac{\mathrm{i} \omega l}{c_{\mathrm{S}}} \cos (\varphi)\left[\frac{\frac{2 P_{1}}{\cos (\varphi)}}{1+\frac{P_{1}}{\cos (\varphi)}}\right]^{2}
$$

In the vicinity of the natural oscillator frequency, this wave is in phase quadrature (neglecting the damping) with the diffracted wave at the zero order. As shown on Fig. 6, its amplitude is directly related to the scale ratio and reach a maximum around the oscillator natural frequency.

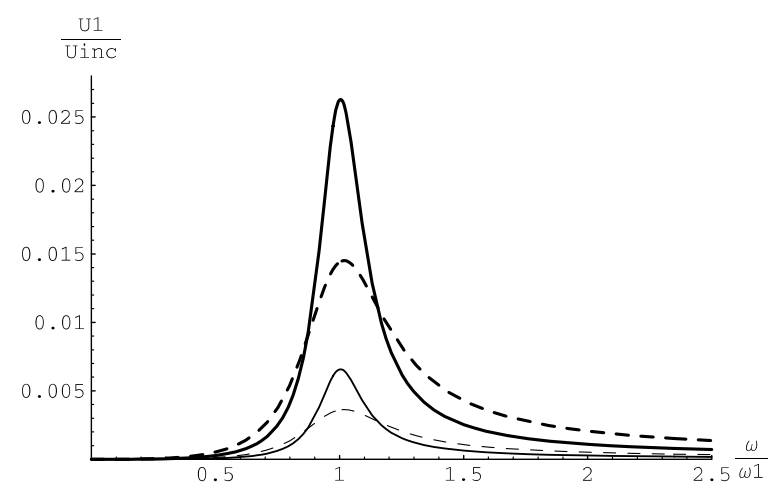

Fig. 6. Amplitude of the first macro-field corrector $\mathbf{U}^{\mathbf{1}}$ according to $\omega / \omega_{1}$ for two $\mathrm{SH}$ incidence angles (plain line: normal incidence, dashed line $\pi / 3$ incidence) and two values of $\epsilon_{1}=\omega_{1} l / c_{\mathrm{S}}\left(\epsilon_{1}=0.2\right.$ : bold lines; $\epsilon_{1}=0.05$ : normal lines $)$. Calculations performed for $\eta_{1} / 2 \xi_{1}=10$, $s=0.5, v=1 / 3$. 


\subsection{Anisotropic resonant surface}

In the case of an isotropic surface, the preceding results apply for any $\mathrm{SH}_{a}$ wave polarised in the direction a inclined of an angle $\alpha$ with the main direction $\mathbf{e}_{1}\left(\mathbf{a}=\cos (\alpha) \mathbf{e}_{1}+\sin (\alpha) \mathbf{e}_{2}\right)$.

If the layer is anisotropic, i.e, $Z_{1} \neq Z_{2}$, it can be shown that a conversion of polarisation arises. For this purpose, suppose for instance that the impedance $Z_{1}$ dominates i.e. $Z_{2}=0$ and consider a vertical incident $\mathrm{SH}_{a}$ wave

$$
\mathbf{U}^{\text {inc }}=U^{i} \exp \left[\mathrm{i} h_{\mathrm{S}} x_{3}\right] \mathbf{a}
$$

The diffracted field is evaluated by applying results (46) to each component in the two main directions

$$
\mathbf{U}^{\mathrm{dif}}=U^{i} \exp \left[-\mathrm{i} h_{\mathrm{S}} x_{3}\right]\left[\frac{1-P_{1}}{1+P_{1}} \cos (\alpha) \mathbf{e}_{1}+\sin (\alpha) \mathbf{e}_{2}\right]=U^{i} \exp \left[-\mathrm{i} h_{\mathrm{S}} x_{3}\right]\left[\frac{1-P_{1} \cos (2 \alpha)}{1+P_{1}} \mathbf{a}+\frac{P_{1} \sin (2 \alpha)}{1+P_{1}} \mathbf{a}_{\perp}\right]
$$

At the natural frequency $\omega_{1}$ a $\pi / 2$-conversion of polarisation from incident $\mathrm{SH}_{a}$ to refracted $\mathrm{SH}_{a \perp}$ $\left(\mathbf{a} \cdot \mathbf{a}_{\perp}=0\right)$ will be observed at angles $\alpha_{0}$ such that

$$
1-\cos \left(2 \alpha_{0}\right) P_{1}\left(\omega_{1}\right)=0 \quad \text { i.e. } \cos \left(2 \alpha_{0}\right)=-2 \xi_{1} / \eta_{1}
$$

With the weak damping assumption, this leads to $\alpha_{0} \approx \pm\left(\pi / 4+\xi_{1} / \eta_{1}\right)$. Thus, around the resonant frequency, such oscillator layer works as a mechanical "depolariser" of $\mathrm{SH}_{\alpha_{0}}$ waves, see Fig. 7.

The corrected fields present only a component polarised according to $\mathbf{e}_{1}$. Their expression are directly derived by multiplying by $\cos (\alpha)$ the expressions obtained in the previous subsection.

In the more general case of oblique incidence, when the polarisation does not coincide with a main direction of the oscillators, the surface force would present three components. Consequently, a mode conversion from $\mathrm{SH}$ wave to $\mathrm{SV}$ and $\mathrm{P}$ wave arises.

\subsection{Refraction of $S V$ and $P$ waves}

Consider now an incident SV wave propagating obliquely in the direction $\mathbf{d}=\sin (\varphi) \mathbf{e}_{1}+\cos (\varphi) \mathbf{e}_{3}$, and polarised in the direction $\mathbf{d}_{\perp}=\cos (\varphi) \mathbf{e}_{1}-\sin (\varphi) \mathbf{e}_{3}$

$$
\left.\mathbf{U}^{\text {inc }}=U^{i} \exp \left[\mathrm{i} h_{\mathrm{S}} \mathbf{d} \cdot \mathbf{x}\right)\right] \mathbf{d}_{\perp}
$$

As $\mathbf{Z}^{0}$ is diagonal in the frame $\left(\mathbf{e}_{i}\right)$, the incident wave induces on $\Gamma$ stress and motion in the plane $\left(\mathbf{e}_{1}, \mathbf{e}_{3}\right)$. Then, to satisfy the Descartes law, the diffracted field is decomposed into down warding SV and $\mathrm{P}$ waves respectively propagating in the directions $\mathbf{d}^{\mathrm{S}}$ and $\mathbf{d}^{\mathrm{P}}$ :

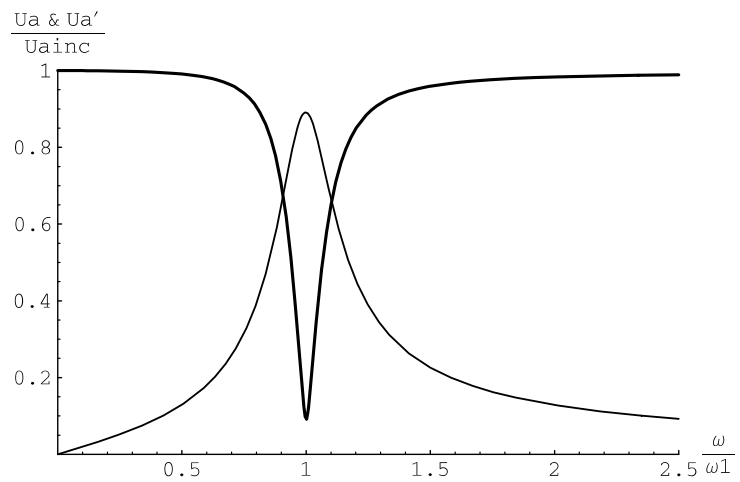

Fig. 7. Depolarisation effect of an anisotropic resonant surface $\left(Z_{2}=0\right)$ according to $\omega / \omega_{1}$ for an $\mathrm{SH}_{\alpha_{0}}$ wave of normal incidence polarised in the direction $\alpha_{0}$ (see text). Bold line: refracted component $\alpha_{0}$-polarised. Normal line: refracted component polarised in the perpendicular direction. Calculations performed for $\eta_{1} / 2 \xi_{1}=10$ corresponding to a polarisation angle $\alpha_{0}=47^{0} 87$. 


$$
\begin{aligned}
& \mathbf{U}^{\mathrm{SV}}=S V \exp \left[\mathrm{i} h_{\mathrm{S}} \mathbf{d}^{\mathrm{S}} \cdot \mathbf{x}\right] \mathbf{d}_{\perp}^{\mathrm{S}} \quad \mathbf{d}^{\mathrm{S}}=\sin (\varphi) \mathbf{e}_{1}-\cos (\varphi) \mathbf{e}_{3} \quad \mathbf{d}_{\perp}^{\mathrm{S}}=\cos (\varphi) \mathbf{e}_{1}+\sin (\varphi) \mathbf{e}_{3} \\
& \mathbf{U}^{\mathrm{P}}=P \exp \left[\mathrm{i} h_{\mathrm{P}} \mathbf{d}^{\mathrm{P}} \cdot \mathbf{x}\right] \mathbf{d}^{\mathrm{P}} \quad \mathbf{d}^{\mathrm{P}}=\sin (\psi) \mathbf{e}_{1}-\cos (\psi) \mathbf{e}_{3} \quad \sin (\psi)=\frac{c_{\mathrm{P}}}{c_{\mathrm{S}}} \sin (\varphi)
\end{aligned}
$$

The boundary condition (44) expressed on matrix form gives two equations involving the impedance of the oscillator layer in both tangential $\left(\mathbf{e}_{1}\right)$ and normal $\left(\mathbf{e}_{3}\right)$ directions:

$$
\begin{aligned}
& \left(U^{i}\left(\mathbf{d} \otimes \mathbf{d}_{\perp}+\mathbf{d}_{\perp} \otimes \mathbf{d}\right)+S V\left(\mathbf{d}^{\mathrm{S}} \otimes \mathbf{d}_{\perp}^{\mathrm{S}}+\mathbf{d}_{\perp}^{\mathrm{S}} \otimes \mathbf{d}^{\mathrm{S}}\right)+P \frac{c_{\mathrm{S}}}{c_{\mathrm{P}}}\left(2 \mathbf{d}^{\mathrm{P}} \otimes \mathbf{d}^{\mathrm{P}}+\frac{\lambda}{\mu} \mathbf{I}\right)\right) \cdot \mathbf{e}_{3} \\
& =\mathbf{P}\left[U^{i} \mathbf{d}_{\perp}+S V \mathbf{d}_{\perp}^{\mathrm{S}}+P \mathbf{d}^{\mathrm{P}}\right] \quad \text { where } \mathbf{P}=-\frac{\mathrm{i} \omega \mathbf{Z}}{\mu \mathrm{i} h_{\mathrm{S}}}
\end{aligned}
$$

Obviously, the SV-P mode conversion is strongly frequency dependant with phase shifts, specially around the natural frequency in both directions.

The analytical resolution in the general case would lead to cumbersome expressions of $S V$ and $P$ and a full analysis is out of the scope of this paper. For this reason we focus on particular cases where the impedance is large in one direction and vanishes in the other. This corresponds to an atypical situation, as "rigid" and free conditions, respectively in the direction of large and negligible impedance, are mixed in the same problem.

If we consider $Z_{1}^{0}$ large and $Z_{3}^{0}=0$, we obtain (the terms $\Phi_{w i}, w=s, p$ involve trigonometric functions of $\varphi$ and $\psi$ ):

$$
S V \approx U^{i}\left[1+\frac{\Phi_{s 1}}{P_{1}}\right] \text { and } P \approx U^{i} \frac{\Phi_{p 1}}{P_{1}}
$$

Inversely the case $Z_{3}^{0}$ large and $Z_{1}^{0}=0$ leads to

$$
S V \approx-U^{i}\left[1+\frac{\Phi_{s 3}}{P_{3}}\right] \text { and } P \approx U^{i} \frac{\Phi_{p 3}}{P_{3}}
$$

Thus in both cases, the converted $\mathrm{P}$ wave is of weak amplitude around the natural frequencies. Further calculation shows that in both cases the total motion polarised in the direction of large impedance tends to vanish. The refracted field is therefore constituted by the almost fully reflected incident mode (with the adequate phase shift), and a weak converted mode. The same approach applied to incident $\mathrm{P}$ wave gives similar results, i.e. a weak conversion. Consequently, if the natural frequencies $\omega_{1}$ and $\omega_{3}$ differ sufficiently, around both resonances the oscillator layer tends to reduce significantly the SV to P, and P to SV mode conversion for a wide range of incidence angle (compared to the free surface case where conversion disappears at normal incidence only). An illustration of this effect is given on Fig. 8, where the comparison with the free surface shows the drastic modification of the diffracted field induced by the resonant surface.

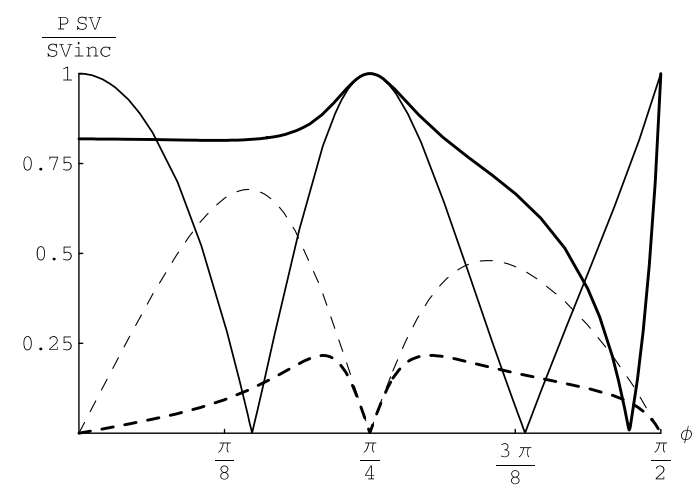

Fig. 8. Reduction of conversion of SV to $\mathrm{P}$ wave $\left(Z_{3}=0\right)$ at the resonant frequency $\left(\omega=\omega_{1}\right)$ according to the incidence of SV wave. Bold plain line: amplitude of the refracted SV wave; bold dashed line: amplitude of the refracted P wave. Normal lines correspond to a free surface (SV plain line, $\mathrm{P}$ dashed line). Calculations performed for $\eta_{1} / 2 \xi_{1}=10, v=1 / 3$. 
The features of the correctors are qualitatively the same as in the previous cases with a more complex geometry. The BL field presents a normal and a tangential component. The surface forces radiating the macro-field take the form (here the non-local source does not cancel out):

$$
\begin{aligned}
\mathrm{i} \omega \mathbf{Y}^{1} \cdot \nabla_{x \alpha} \mathbf{U}^{0} & =\lambda \tilde{A}_{3} \mathrm{i} \omega Z_{3}^{0} \mathrm{i} h_{\mathrm{S}} \sin (\varphi) U_{3}^{0} \mathbf{e}_{1}+\mu \tilde{A}_{1} \mathrm{i} \omega Z_{1}^{0} \mathrm{i} h_{\mathrm{S}} \cos (\varphi) U_{1}^{0} \mathbf{e}_{3}-\omega^{2} \mathbf{Z}^{1} \cdot \mathbf{U}^{0} \\
& =\left(\mathrm{i} \omega Z_{1}^{0}\right)^{2} B_{1} U_{1}^{0} \mathbf{e}_{1}+\left(\mathrm{i} \omega Z_{3}^{0}\right)^{2} B_{3} U_{3}^{0} \mathbf{e}_{1}
\end{aligned}
$$

Therefore, $\mathbf{U}^{1}$ is constituted of two diffracted $\mathrm{P}$ and $\mathrm{S}$ plane waves respecting the Descartes law with an amplitude of the order of the scale ratio around the natural frequency.

\section{Limit of validity of the modelling}

Let us now come back to the limitations of the proposed modelling. The main restriction lies in the frequency range of validity, related to the scale separation hypothesis. To investigate this point consider for instance the response to an oblique incident $\mathrm{SH}$ wave. By spatial Fourier transform, the quasi-periodic stress may be split up into a discrete series (assuming a square period $l \times l)$ modulated by the horizontal wavelength (in the case of normal incidence a strict periodicity is recovered):

$$
\mathbf{t}=\left(\sum_{m, n=-\infty}^{\infty} \mathbf{t}_{\varphi}^{m, n} \exp \left[(2 \mathrm{i} \pi m / l) x_{1}\right] \exp \left[(2 \mathrm{i} \pi n / l) x_{2}\right]\right) \exp \left[\mathrm{i} h_{\mathrm{S}} \sin (\varphi) x_{1}\right] \quad \text { with } m, n \text { integers }
$$

Using the argument of the Descartes law for each term, the wave field inside the isotropic elastic medium is constituted by discrete series of $\mathrm{S}$ and $\mathrm{P}$ waves in the form $(\mathrm{W}=\mathrm{S}$ or $\mathrm{P})$ :

$$
\mathbf{W}=\sum_{m, n=-\infty}^{\infty} \mathbf{W}_{\varphi}^{m, n} \exp \left[\left(\mathrm{i} h_{\mathrm{S}} \sin (\varphi)+2 \mathrm{i} \pi m / l\right) x_{1}\right] \exp \left[(2 \mathrm{i} \pi n / l) x_{2}\right] \exp \left[\mathrm{i} \xi_{m, n}^{\mathrm{W}} h_{\mathrm{S}} x_{3}\right]
$$

with

$$
\left(h_{\mathrm{S}} \sin (\varphi)+m 2 \pi / l\right)^{2}+(n 2 \pi / l)^{2}+\left(h_{m n}^{\mathrm{W}}\right)^{2}=\left(\omega / c_{\mathrm{W}}\right)^{2}=\left(2 \pi / \lambda_{\mathrm{W}}\right)^{2}
$$

that is, using $\epsilon=\omega l / c_{\mathrm{S}}$ :

$$
[\sin (\varphi)+m 2 \pi / \epsilon]^{2}+[n 2 \pi / \epsilon]^{2}+\left[\xi_{m, n}^{\mathrm{W}}\right]^{2}=\left[c_{\mathrm{S}} / c_{\mathrm{W}}\right]^{2}
$$

The terms $m=n=0$ correspond to a $\mathrm{P}$ and a $\mathrm{S}$ wave propagating in the direction defined by the Descartes law applied to the incident $S$ wave. By construction, their amplitudes are directly related to the term $\mathbf{t}_{\varphi}^{0,0}$ of (58), i.e. the average value of $\mathbf{t}$ on the period. The nature of the next term depends on the $\epsilon$ value:

- if $\epsilon<\pi$, that defines a frequency range including the scale separation range, then, whatever $\varphi,\left(\xi_{m n}^{\mathrm{W}}\right)^{2}<0$ for any $m, n, m n \neq 0$ : these inhomogeneous waves vanish as the depth increases. Then, the far diffracted field only consists of the $(m n=0)$ waves resulting from the mean loading on the surface. This is fully consistent with the boundary layer approach (including high orders), the whole inhomogeneous waves standing for the boundary layer, and the single $\mathrm{S}$ and $\mathrm{P}$ homogeneous wave for the far field.

- if $\epsilon>2 \pi$, that is when the scale separation is not satisfied, in addition to the $(m n=0)$ waves, a finite number of homogeneous waves (such that $\left(h_{m n}^{\mathrm{W}}\right)^{2}>0$ ) propagate in discrete directions and must be integrated in the far field. As their amplitudes depend on the terms $\mathbf{t}_{\varphi}^{m, n}, m n \neq 0$ of (58) the single knowledge of the average loading is not sufficient to determine the far field. The periodicity imposed on the surface is transmitted to the far diffracted field. This is in contradiction with the boundary layer assumption (i.e. vanishing small scale variations far from $\Gamma$ ) and the homogenised approach breaks down.

Such arguments, independent of the resonant surface properties, can be extended with slight modifications to other geometry of the period. Without giving a complete justification, it tends to prove that the range of validity initially defined by the scale separation expressed in the form, $l \ll \lambda / 2 \pi$ may be extended (including higher orders in the description) to a less restrictive domain, i.e. $l<\lambda / 2 \pi$. 
Note that the key point of the reasoning (i.e. homogeneous or inhomogeneous waves) is not strictly related to the periodicity. In fact, if the stress distribution takes the more general non-quasi-periodic form:

$$
\mathbf{t}=\left(\mathbf{t}_{\varphi}(0,0)+\int_{|u|>1 ;|v|>1} \mathbf{t}_{\varphi}(u, v) \exp \left[(2 \mathrm{i} \pi u / l) x_{1}\right] \exp \left[(2 \mathrm{i} \pi v / l) x_{2}\right] \mathrm{d} u \mathrm{~d} v\right) \exp \left[\mathrm{i} h_{\mathrm{S}} \sin (\varphi) x_{1}\right]
$$

i.e. if the local variations appear at a scale of the order (and smaller) than $l$, the same kind of conclusions continues to hold for the scale separation frequency range. This lead us to think that the second restriction i.e. the periodicity assumption may be relaxed. Very likely, a non-periodic distribution presenting a spatial invariance at a scale $l$ (in other word resulting from an elementary representative surface), would lead to phenomena of the same nature if the scale separation is satisfied. Particularly, it may be inferred that the notion of BL field may be extended in a similar form by considering a mesoscopic surface (of the order of $l / \sqrt{\epsilon}$ ) that permits to cancel out statistically the non-periodic $l$-scale variations at the border. The high order terms should also follow the Descartes law, the other contributions to the field being inhomogeneous. It is also worth mentioning that results presented in this paper take a similar form that results established on the random assumption by Lombaert et al. [10].

Inversely, at shorter wavelengths, diffraction phenomena are very different in ordered and disordered media and the descriptions significantly differ. Finally, for a fully disordered surface (if any), the stress would present variations at any scale so that the notion of scale separation disappears and any homogenisation method (fuzzy structure approach included) is irrelevant to describe the continuous spectrum of the diffracted field.

\section{Conclusion}

Within the frame of homogenisation methods, the effective boundary conditions adapted to quasi-periodic forces have been established. The natural macroscopic condition given by the average equilibrium on the periodic surface is recovered at the leading order, independently of the properties of the medium. It is shown that, at the next order, a non-local term associated to the macro-gradient of the surface force distribution and involving the medium elasticity must be taken into account. These results, established for forces harmonically oscillating in time, are also valid in the static case. They prove that the non-local concepts mainly used when studying heterogenous medium should also be considered for boundary conditions.

From this approach, it is demonstrated that the description of an oscillator layer by an equivalent surface impedance corresponds to the first order approximation. For poor scale separation, a local and a non-local corrector of the impedance must be added. This provides a improvement to the impedance-type boundary condition used in the "fuzzy structure" and deterministic approaches [6]. This also gives a justification by defining the effective range of validity of these modelling. One may consider that for practical application the relevant frequency range is such that $l<\lambda / 2 \pi$.

As for wave propagation, the present paper shows that, owing to the resonance, the perturbations by an oscillator layer may be of the leading order even for light oscillating masses, and induce a de-amplification associated to a memory effect. The main parameters that govern the phenomena are given and assessed. Some non-classical features, such as modes conversion, frequency band of efficiency, effect of anisotropic layer, and corrector fields are described. The method enables to consider more complex situations than those presented in the paper, i.e. several oscillators of different natural frequencies and orientations, oscillators presenting several modes or other homogeneous and inhomogeneous, plane, cylindrical, spherical waves fields including surface waves, provided that the scale separation is respected.

As a concluding example, consider a very corrugated surface obtained by making parallel and periodically spaced slots of depth $H$ on the surface of an elastic medium.

If the slots are realised in a single direction, we get series of parallel "walls" of thickness $e$, height $H$, reproduced every $l$, Fig. 9. Slots in the two perpendicular directions give on the top surface a periodic distribution of square "beams" of section $S=e^{2}$, height $H$, located each $l \times l$, Fig. 10 .

In the three directions, when focusing on the first vibration mode of the surface elements, each of them can be modelised as a single degree of freedom oscillator whose mass is the modal mass. This enables to define one anisotropic and one isotropic resonant surface whose characteristics are presented in Tables 1 and 2 in 


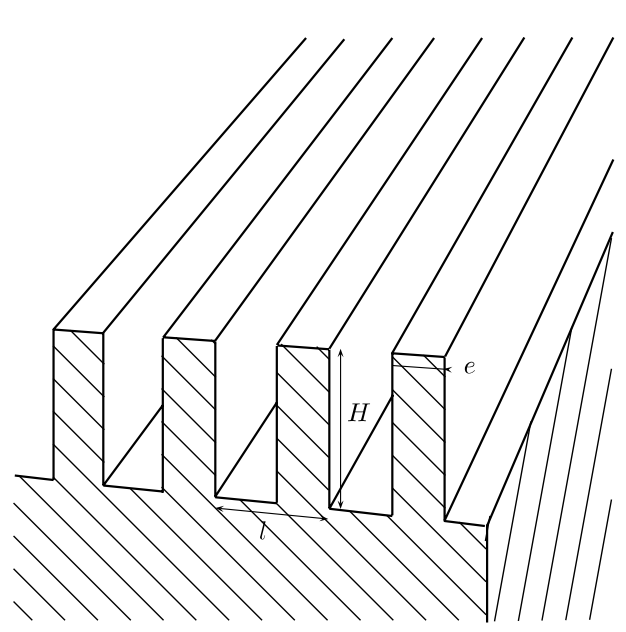

Fig. 9. Anisotropic resonant surface made of a series of parallel "walls" of thickness $e$, height $H$, reproduced every $l$.

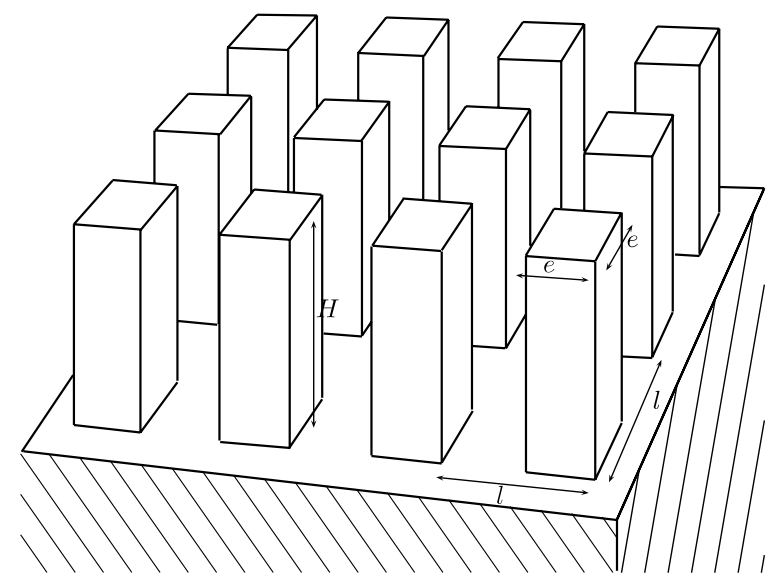

Fig. 10. Isotropic resonant surface made of a series of beams of section $S=e^{2}$, height $H$, located each $l \times l$.

Table 1

Characteristic parameters of an anisotropic resonant surface made of a parallels slots drawn in the direction $\mathbf{e}_{1}$ on the surface of an elastic medium, see Fig. 9

\begin{tabular}{|c|c|c|c|}
\hline Direction & $\mathbf{e}_{1}$ & $\mathbf{e}_{2}$ & $\mathbf{e}_{3}$ \\
\hline Vibration mode & Shear & Out of plane bending & Compression \\
\hline \multirow[t]{2}{*}{ First eigen frequency } & $\omega_{1}=\frac{\pi}{2 H} \sqrt{\frac{\mu}{\rho}}$ & $\omega_{2}=\left(\frac{\pi \gamma}{2 H}\right)^{2} \sqrt{\frac{E^{\prime} I}{\rho S}}$ & $\omega_{3}=\frac{\pi}{2 H} \sqrt{\frac{E^{\prime}}{\rho}}$ \\
\hline & & $\gamma \approx 1.195 ; \frac{I}{S}=\frac{e^{2}}{12}$ & $E^{\prime}=\frac{2 \mu}{1-v}$ \\
\hline Modal mass/mass & $8 / \pi^{2}$ & $\beta \approx 0.613$ & $8 / \pi^{2}$ \\
\hline$\eta_{i}$ & $\eta_{1}=\frac{4}{\pi} \frac{e}{l}$ & $\eta_{2}=\beta\left(\frac{\pi \gamma}{2}\right)^{2} \sqrt{\frac{1}{6(1-v)}} \frac{e}{H} \frac{e}{l}$ & $\eta_{3}=\frac{4}{\pi} \frac{\sqrt{1-2 v}}{1-v} \frac{e}{l}$ \\
\hline$\epsilon\left(\omega_{i}\right)$ & $\epsilon_{1}=\frac{\pi}{2} \frac{l}{H}$ & $\epsilon_{2}=\left(\frac{\pi \gamma}{2}\right)^{2} \sqrt{\frac{1}{6(1-v)}} \frac{e}{H} \frac{l}{H}$ & $\epsilon_{3}=\frac{\pi}{2} \frac{\sqrt{1-2 v}}{1-v} \frac{l}{H}$ \\
\hline
\end{tabular}

function of the geometric parameters and the mechanical properties of the medium. According to the requirements (48) and (49), significant effects will be observed around the eigen frequencies if, for instance, $e / l=\mathrm{O}(1)$ and $e / H \approx 0.1$. The isotropic layer whose horizontal and vertical frequency are very different will be favorable to the reduction of P-S conversion (as described in Section 5.3), while the depolarisation of SH waves (as 
Table 2

Characteristic parameters of an isotropic resonant surface made of a periodic pattern of square beams of the same material than the elastic medium, see Fig. 10

\begin{tabular}{lll}
\hline Direction & $\mathbf{e}_{1}, \mathbf{e}_{2}$ & $\mathbf{e}_{3}$ \\
Vibration mode & Bending & Compression \\
First eigen frequency & $\omega_{1}=\omega_{2}=\left(\frac{\pi \gamma}{2 H}\right)^{2} \sqrt{\frac{E I}{\rho S}}$ & $\omega_{3}=\frac{\pi}{2 H} \sqrt{\frac{E}{\rho}}$ \\
& $\gamma \approx 1.195 ; \frac{I}{S}=\frac{e^{2}}{12}$ & $E=2 \mu(1+v)$ \\
Modal mass/mass & $\beta \approx 0.613$ & $8 / \pi^{2}$ \\
$\eta_{i}$ & $\eta_{1}=\eta_{2}=\beta\left(\frac{\pi \gamma}{2}\right)^{2} \sqrt{\frac{1+v}{6}} \frac{e}{H}\left(\frac{e}{l}\right)^{2}$ & $\eta_{3}=\frac{4}{\pi} \sqrt{\frac{(1+v)(1-2 v)}{1-v}}\left(\frac{e}{l}\right)^{2}$ \\
$\epsilon\left(\omega_{i}\right)$ & $\epsilon_{1}=\epsilon_{2}=\left(\frac{\pi \gamma}{2}\right)^{2} \sqrt{\frac{1+v}{6}} \frac{e}{H} \frac{l}{H}$ & $\epsilon_{3}=\frac{\pi}{2} \sqrt{\frac{(1+v)(1-2 v)}{1-v}} \frac{l}{H}$ \\
\hline
\end{tabular}

described in Section 5.2) will be observed with the anisotropic layer. Number of variations can be contemplated from this simple example, which shows that resonant top layers can be designed rather simply in order to control at a given frequency the refracted wave field.

\section{Acknowledgement}

This research was supported by the French CNRS program ACI-CATNAT.

\section{Appendix 1. Boundary layer field in the case of rigid basement}

In fact, a full description of the oscillator behaviour should also include the base rotation $\psi$ and the transmitted momentum M. However, as the leading motions and macro-stresses only depend on the macro-variables, they are not able to describe neither rotation nor momentum which necessarily imply local variations. Therefore, this complementary part of the behaviour only arises at the next order and is balanced by the BL field. Hence, the consistency with the two scales expansions imposes to write the generalised impedance relations on the form (assuming negligible the momentum-translation and force-rotation coupling impedance matrix)

$$
\left[\begin{array}{c}
\mathbf{R} \\
\mathbf{M}
\end{array}\right]=\mathrm{i} \omega\left[\begin{array}{cc}
\mathbf{Z} & 0 \\
0 & 0
\end{array}\right] \cdot\left[\begin{array}{c}
\mathbf{u}_{\mathbf{S}} \\
\psi
\end{array}\right]+\epsilon \mathrm{i} \omega\left[\begin{array}{cc}
0 & 0 \\
0 & \mathbf{Z}_{\psi}
\end{array}\right] \cdot\left[\begin{array}{c}
\mathbf{u}_{\mathbf{S}} \\
\psi
\end{array}\right]
$$

and, expanding the base motions:

$$
\mathbf{R}^{\mathbf{0}}=\mathrm{i} \omega \mathbf{Z} \cdot \mathbf{U}_{\mathbf{S}}^{\mathbf{0}}, \quad \text { and } \quad\left[\begin{array}{c}
\mathbf{R}^{\mathbf{1}} \\
\mathbf{M}^{\mathbf{1}}
\end{array}\right]=\mathrm{i} \omega\left[\begin{array}{c}
\mathbf{Z} \mathbf{U}_{\mathbf{S}}^{\mathbf{1}} \\
\mathbf{Z}_{\psi} \Psi^{\mathbf{0}}
\end{array}\right]
$$

Consequently, the behaviour defined by the impedance matrix restricted to forces and translation motions (33) is sufficient for the leading order analysis, while the global impedance matrix is needed for the determination of the BL field.

In presence of a rigid base, the BL field is governed by the same equations as previously (15), (17), (18), (9), except for the condition (16) on $\Sigma_{0}$ that must now be decomposed in three parts:

(i) outside $S$, the surface force $\mathbf{t}^{0}$ vanishes,

$$
\mathbf{t}^{0}=-\mathbf{C}:\left[\mathbf{e}_{\mathbf{y}}\left(\mathbf{u}^{\star 1}\right)+\mathbf{e}_{\mathbf{x}}\left(\mathbf{u}^{\mathbf{0}}\right)\right] \cdot \mathbf{e}_{\mathbf{3}}=\mathbf{0}
$$

(ii) on $S$, the medium follows the rigid base motion,

$$
\mathbf{u}^{\star 1}=\overline{\mathbf{u}}^{\star 1}+\mathbf{y} \times \Psi^{\mathbf{0}} \quad \text { unknown rigid body motion }
$$


(iii) moreover, the surface force $\mathbf{t}^{0}$ balances the resultant force and the momentum:

$$
\begin{aligned}
& \int_{S} \mathbf{t}^{\mathbf{0}} \mathrm{d} s=-\int_{S} \mathbf{C}:\left[\mathbf{e}_{\mathbf{y}}\left(\mathbf{u}^{\star \mathbf{1}}\right)+\mathbf{e}_{\mathbf{x}}\left(\mathbf{u}^{\mathbf{0}}\right)\right] \cdot \mathbf{e}_{3} \mathrm{~d} s=\mathbf{R}^{0}=\Sigma_{0} \mathbf{T}^{\mathbf{0}} \\
& \int_{S} \mathbf{t}^{0} \times \mathbf{y} \mathrm{d} s=-\int_{S} \mathbf{C}:\left[\mathbf{e}_{\mathbf{y}}\left(\mathbf{u}^{\star \mathbf{1}}\right)\right] \cdot \mathbf{e}_{\mathbf{3}} \times \mathbf{y} \mathrm{d} s=\mathbf{M}^{1}=\mathbf{Z}_{\psi} \Psi^{\mathbf{0}}
\end{aligned}
$$

It is clear that the solution of this linear problem only depends on the single forcing term $\mathbf{T}^{\mathbf{0}}$. Thus, as asserted, the BL field takes the same form, where now a depends on $\mathbf{Z}_{\psi}$

$$
\mathbf{u}^{\star 1}\left(\mathbf{x}_{\alpha}, \mathbf{y}\right)=\mathbf{a}^{\mathbf{i}}(\mathbf{y}) \mathbf{T}_{\mathbf{i}}^{\mathbf{0}}\left(\mathbf{x}_{\alpha}\right)
$$

and consequently the translation and rotation defining the rigid base motion read:

$$
\overline{\mathbf{u}}^{\star 1}\left(\mathbf{x}_{\alpha}\right)=\mathbf{b}^{\mathrm{i}} \mathbf{T}_{\mathbf{i}}^{\mathbf{0}}\left(\mathbf{x}_{\alpha}\right), \quad \text { and } \quad \Psi^{\mathbf{0}}\left(\mathbf{x}_{\alpha}\right)=\Phi^{\mathrm{i}} \mathbf{T}_{\mathbf{i}}^{\mathbf{0}}\left(\mathbf{x}_{\alpha}\right)
$$

\section{Appendix 2. Approximated boundary layer field}

Here is a proposal to construct an approximated boundary layer field adapted to tooth-like stress distribution corresponding to one oscillator of surface $S=s \cdot \Sigma_{0}$ in the period. The idea is to follow the main requirements identified by homogenisation (elasto-static problem, zero mean force on the top surface, zero stress and displacement on the bottom, periodicity and "thickness" of the order of the period) using the concept of conical stress diffusion widely used in engineering sciences (see for instance [19]).

Consider a rigid circular plate of surface $S$ and radius $r_{0}$ loaded by a static force $\mathbf{R}$, resting on the top surface of an elatic half-space. The cone approximation (denoted by ${ }^{c}$ ) is based on the following simplifying assumptions:

- the stresses are confined in a truncated cone $C$ of vertical axis passing through the plate center,

- the cone appex located at the high $h=\beta r_{0}$ above the medium is determined by equating the exact plate-onmedium rigidity and cone model rigidity,

- only the components and the stress balance in the direction of the loading are considered, and the variables depend only on the depth (here denoted by $z$ ).

Assume an horizontal force $\mathbf{R}=\mathbf{R e}_{\mathbf{1}}$ (vertical force can be treated similarly). In the loading direction, the balance of forces applied to a thin cone section reads

$$
\frac{\mathrm{d}}{\mathrm{d} z}\left[\tau^{c}(z) S^{c}(z)\right]=0
$$

where $S^{c}(z)=\left(\frac{z+h}{h}\right)^{2} S$ is the cone section at the depth $z$, and, according to the elastic behaviour, the shear stress $\tau^{c}$ and motion $u^{c}$ are related by:

$$
\tau^{c}(z)=\mu \frac{\mathrm{d}}{\mathrm{d} z} U^{c}(z)
$$

Integrating (67), (68) and disregarding rigid motion gives the cone shaped field defined on $C$ only:

$$
\tau^{c}(z)=\tau_{0}^{c}\left(\frac{h}{z+h}\right)^{2} \quad U^{c}(z)=-\frac{\tau_{0}^{c} h}{\mu}\left(\frac{h}{z+h}\right)=U_{0}^{c}\left(\frac{h}{z+h}\right)
$$

The force $R=T^{0} \Sigma_{0}$ is balanced by the stress on the surface and is related to the base displacement $U_{0}^{c}$ by the exact plate-on-medium rigidity

$$
R=-\pi r_{0}^{2} \tau_{0}^{c}=U_{0}^{c} \mu \frac{\pi r_{0}^{2}}{h}, \quad \text { and } \quad R=U_{0}^{c} \mu \frac{8 r_{0}}{2-v}
$$


so that

$$
\beta=\frac{h}{r_{0}}=\frac{\pi(2-v)}{8}
$$

Consider now a $\Sigma_{0}$-periodic distribution of such plates identically loaded. The cone shaped field diffused by each plate cover the whole medium surface at a depth $H$ such that

$$
S^{c}(H)=\left(\frac{H+h}{h}\right)^{2} S=\Sigma_{0} \quad \text { i.e. } H=(1 / \sqrt{s}-1) h
$$

By construction $H$ defines the boundary layer thickness estimate, and at this level the displacement and stress can be looked as uniform in a first approximation (disregarding un- and over-lapped zones) and given by

$$
\tau^{c}(H)=\tau_{0}^{c}\left(\frac{h}{H+h}\right)^{2}=\tau_{0}^{c} s=-R / \Sigma_{0}=T^{0} \quad U^{c}(H)=-\frac{\tau_{0}^{c} h}{\mu}\left(\frac{h}{H+h}\right)
$$

To satisfy the BL requirements, add to this field an homogeneous field, $U^{h}$, in the layer (i.e. inside and outside the cone $C$ ) defined in such a way that the total stress and displacement cancel out on $z=H$.

$$
U^{h}(z)=-\left[(z-H) \frac{\tau^{c}(H)}{\mu}+U^{c}(H)\right]
$$

The field $U^{\star} \mathbf{e}_{\mathbf{1}}=\left(U^{c}+U^{h}\right) \mathbf{e}_{\mathbf{1}}\left(U^{c}\right.$ is set to zero outside $C$ ) follows all the aforementioned requirements for an approximated BL layer field. On the top surface $\Gamma$, one obtains

$$
\begin{aligned}
& \text { on } S \quad U^{\star}=-\frac{T^{0}}{\mu} \frac{h}{s}(1-\sqrt{s})^{2}, \quad \tau^{\star}=T^{0} \frac{1-s}{s} \\
& \text { on } \Sigma_{0}-S \quad U^{\star}=\frac{T^{0}}{\mu} \frac{h}{s}\left[1-(1-\sqrt{s})^{2}\right], \quad \tau^{\star}=-T^{0}
\end{aligned}
$$

Calculations are very similar for vertical force. The differences come from the cone aspect ratio that takes the value $\beta^{\prime}=\pi / 2$ and the normal stress-strain relation that reads in the cone confined by the surrounding medium $\sigma_{c}(z)=2 \mu /(1-v) \frac{\mathrm{d}}{\mathrm{d} z} U^{c}(z)$. The approximated BL field $V^{\star} \mathbf{e}_{\mathbf{3}}$ associated to a vertical mean stress $T_{3}^{0} \mathbf{e}_{3}$ takes the following values on the top surface $\Gamma$ :

$$
\begin{aligned}
& \text { on } S \quad V^{\star}=-\frac{T_{3}^{0}}{2 \mu} \frac{(1-v) h}{s}\left(1-\sqrt{s}\left[1+\frac{1-2 v}{(1-v)^{2}}(1-\sqrt{s})\right]\right) \\
& \text { on } \Sigma_{0}-S \quad V^{\star}=\frac{T_{3}^{0}}{2 \mu} \frac{(1-v) h}{s} \sqrt{s}\left[1+\frac{1-2 v}{(1-v)^{2}}(1-\sqrt{s})\right]
\end{aligned}
$$

Simple estimates of tensors (all diagonal) A, $\underline{\mathbf{A}}$ and $\mathbf{B}$ derived from those expressions are given in Section 4.3. Note that only the elastic properties of the medium and the surface density of oscillator basis are involved. The local organisation of the oscillators that influences the exact solution disappear in this approximation. One can refer to Boutin and Roussillon [6] for a generalisation of this approach treating more complex cases including different oscillators.

\section{References}

[1] J.L. Auriault, Heterogeneous medium. Is an equivalent macroscopic description possible?, Int J. Engng. Sci. 29 (7) (1991) $785-795$.

[2] A. Bensoussan, J.L. Lions, G. Papanicolaou, Asymptotic Methods in Periodic Structures, North-Holland, Amsterdam, 1978.

[3] D.H. Berman, J.S. Perkins, Rayleigh method for scattering from random and deterministic interfaces, J. Acoust. Soc. Am. 88 (1990) 1032-1044.

[4] G. Bouchitté, A. Lidouh, P. Suquet, Homogeneisation de frontiere pour la modelisation du contact entre un corps deformable nonlineaire et un corps rigide, C. R. Acad. Sci. Paris 313 (1991) 967-972.

[5] C. Boutin, J.L. Auriault, Rayleigh scattering in elastic composite materials, Int. J. Engng. Sci. 31 (12) (1993) $1669-1689$.

[6] C. Boutin, P. Roussillon, Assessment of the urbanization effect on seismic response, Bull. Seism. Soc. Am. 94 (1) (2004) $251-268$. 
[7] D. Clouteau, D. Aubry, Modification of ground motion in dense urban areas, J. Comput. Acoust. 6 (2001) $1659-1675$.

[8] P. Guéguen, P.Y. Bard, F.J. Chavez-Garcia, Site-City seismic interaction in Mexico City like environment: an analytic study, Bull. Seism. Soc. Am. 92 (2002) 794-804.

[9] C.L. Holloway, E.F. Kuester, SImpedance-type boundary condition for a periodic interface between a dielectric and a highly conducting medium, IEEE Trans. Anten. Prop. 48 (2000) 1660-1672.

[10] G. Lombaert, D. Clouteau, O. Ishizawa, N. Mezher, The city-site effect: a fuzzy substructure approach and numerical simulations, in: Proceedings of the 11th International Conference on Soil Dynamics and Earthquake Engineering, Berkeley, USA, January 2004.

[11] J. Nevard, J.B. Keller, Homogenisation of rough boundary and interfaces, SIAM J. Appl. Math. 57 (1997) $1660-1686$.

[12] E. Sanchez-Palencia, Non-homogeneous media and vibration theory. Lecture Note in Physics, vol. 127, Springer-Verlag, Berlin, 1980.

[13] E.J. Sabina, J.R. Willis, Scattering of SH waves by a rough half-space of arbitrary slope, Geophys. J. Roy. Astron. Soc. 42 (1975) 685-703.

[14] C. Soize, A model and numerical method in the medium frequency range for vibroacoustic predictions using theory of structural fuzzy, J. Acoust. Soc. Am. 94 (2) (1993) 849-865.

[15] M. Strasberg, D. Feit, Vibration damping of large structures induced by attached small resonant structures, J. Acoust. Soc. Am. 99 (21) (1996) 335-344.

[16] C. Tsogka, A. Wirgin, Simulation of seismic response in an idealized city, Soil Dyn. Earth. Engng. 23 (2003) $391-402$.

[17] R.L. Weaver, Multiple-scattering theory for mean responses in a plate with sprung masses, J. Acoust. Soc. Am. 101 (6) (1997) 34663474 .

[18] A. Wirgin, P.Y. Bard, Effects of buildings on the duration and amplitude of ground motion in Mexico city, Bull. Seism. Soc. Am. 86 (3) (1996) 914-920.

[19] J.P. Wolf, Vibration Analysis Using Simple Physical Models, Prentice-Hall, Englewood Cliffs, 1994. 